\title{
MULTILEVEL PRECONDITIONING FOR THE FINITE VOLUME METHOD
}

\author{
YONGHAI LI, SHI SHU, YUESHENG XU, AND QINGSONG ZOU
}

\begin{abstract}
We consider the precondition of linear systems which resulted from the finite volume method (FVM) for elliptic boundary value problems. With the help of the interpolation operator from the trial space to the test space of the FVM and the operator induced by the FVM bilinear form, we show that both wavelet preconditioners and multilevel preconditioners designed originally for the finite element method (FEM) of a boundary value problem can be used to precondition the FVM of the same boundary value problem. We prove that such preconditioners ensure that the resulting coefficient matrix of the FVM has a uniformly bounded condition number. We present seven numerical examples to confirm our theoretical findings.
\end{abstract}

\section{INTRODUCTION}

The finite volume method (FVM) has been intensively studied for several decades. An incomplete list of references for the method includes [1, 3, 4, 6, 7, 9, 10, 11, 12, 21, 26, 27, 28, 29, 30, 31, 36, 37, 48, 49] and the references cited therein. Because of its local conservation of certain physical quantities and its convenience in numerical implementation, it has been widely used in engineering computation. Similarly to the traditional finite element method (FEM), the FVM suffers from the ill-condition of its coefficient matrix. As a result, the numerical solution of the resulting linear system is not stable. To efficiently solve the resulting linear system of FVM, it is crucial to use a preconditioning technique. The main purpose of this paper is to present multilevel preconditioning schemes for the FVM for solving boundary value problems of elliptic partial differential equations of the second order.

The traditional finite element method for solving differential equations has an obvious shortcoming. Its coefficient matrices are not well-conditioned. Preconditioning for such matrices received considerable attention in the last two decades.

Received by the editor July 14, 2010 and, in revised form, April 18, 2011.

2010 Mathematics Subject Classification. Primary 65N08, 65F08, 65N55.

Key words and phrases. The finite volume method, preconditioning, the multilevel method.

The first author was supported by the '985' programme of Jilin University, the National Natural Science Foundation of China (No.10971082) and the NSAF of China (Grant No.11076014).

The second author was partially supported by the NSFC Key Project (Grant No.11031006) and the Provincial Natural Science Foundation of China (Grant No.10JJ7001), and the Aid Program for Science and Technology Innovative Research Team in Higher Educational Institutions of Hunan Province of China.

The third and corresponding author is partially supported by the US Air Force Office of Scientific Research under grant FA9550-09-1-0511, by Guangdong Provincial Government of China through the "Computational Science Innovative Research Team" program, and by the Natural Science Foundation of China under grant 11071286.

The fourth author is supported in part by the Fundamental Research Funds for the Central Universities and National Natural Science Foundation of China under grant 11171359.

(c)2012 American Mathematical Society Reverts to public domain 28 years from publication 
For example, the hierarchical preconditioner was proposed in [46, 47]. The BPX multilevel preconditioner was introduced in [2, 40]. The wavelet preconditioners were studied in [15, 16, 33]. For other aspects of the wavelet method in solving differential equations, see [8, 17, 18, 44, 45] and the references cited therein. Both BPX preconditioner and wavelet preconditioner lead to matrices with uniformly bounded condition numbers.

The basic idea of this paper is to use the wavelet preconditioners developed in [15] and the multilevel preconditioners developed in [2, 38, originally for the Galerkin FEM to precondition the FVM for the same boundary value problem. It is known that the FVM is different from the Galerkin FEM in its test space. In the Galerkin FEM, the test space is the same as the trial space, while in the FVM, the test space is spanned by piecewise polynomials of a lower order with no global continuity requirement. Such a construction of the test space increases flexibility and convenience in computation. Noticing that the trial space and the test space have the same dimensions, a one-to-one mapping was originally introduced in [27] from the trial space to test space. With this mapping, we shall show that a change of basis in the trial space only is sufficient to precondition the FVM schemes. The main purpose of this paper is to develop a general theoretical base for this preconditioning and to confirm theoretical results numerically for several examples of practical importance.

Due to the construction in the trial space and the test space, in general the resulting FVM matrix is nonsymmetric. In this paper, we suggest that the GMRES algorithm be used to solve the corresponding nonsymmetric FVM linear system. For this reason, we also consider the preconditioning of the GMRES algorithm for solving the nonsymmetric linear system resulted from the FVM.

This paper is organized in six sections. In Section 2, we describe the finite volume method for elliptic equations of the second order. In Section 3, we precondition the operator induced by the FVM bilinear form, while in Section 4, we precondition the corresponding finite volume matrix. We present two types of preconditioners. One is obtained by a change basis matrix from the nodal basis to a wavelet (or pre-wavelet) basis. The other one is obtained by using the BPX preconditioning technique presented in [2, 38, 40]. The uniform boundedness of the condition numbers of the preconditioned operator and the preconditioned matrix is shown for both methods. In Section 5, we precondition the FVM when the linear element is used for the elliptic equation having discontinuous coefficients. We prove that in this special case the form of the FVM is equivalent to that of FEM, independent of the jumps in the coefficient. Therefore, the efficient preconditioners for the linear FEM are also efficient for the linear FVM. In Sections 6, we consider special cases of the FVM and present seven examples to confirm the theoretical results concerning the preconditioning. Cases one and six are for the two point boundary value problem. Cases two, three and four are concerned with the Laplace equation using linear elements on rectangle grids, linear elements on triangle grids, and quadratic elements on triangular grids, respectively. Cases five and seven consider, respectively, the elliptic equation with discontinuous coefficients and the Laplace equation using a special quadratic FVM scheme constructed recently in [5]. The numerical results presented in this section confirm the uniform boundedness of the condition numbers of the preconditioned matrices. 


\section{The Finite Volume Method}

In this section, we set up a theoretical setting of the FVM for solving elliptic boundary value problems. Let $d$ be a fixed positive integer and $\Omega \subset \mathbf{R}^{d}$ a bounded open convex polyhedral with boundary $\partial \Omega$. Let $A(x), x \in \Omega$, be a $d \times d$ symmetric matrix whose entries are functions on $\Omega$. We assume that the matrix $A$ satisfies the uniformly elliptic condition, that is, there exists a constant $r>0$ such that

$$
\xi^{T} A(x) \xi \geq r \xi^{T} \xi, \text { for all } x \in \Omega \text { and } \xi \in \mathbf{R}^{d} .
$$

We define an elliptic partial differential operator of the second order by

$$
L u:=-\nabla \cdot(A(x) \nabla u),
$$

and for a given function $f \in L^{2}(\Omega)$ we consider the boundary value problem

$$
\begin{aligned}
& L u=f \text { in } \Omega, \\
& u=0 \text { on } \partial \Omega .
\end{aligned}
$$

For the purpose of constructing the trial space and the test space for the FVM, we now present a primary partition and its corresponding dual partition of the domain $\Omega$. For $n \in \mathbf{N}_{0}:=\{0,1, \ldots\}$, we let $T_{n}$ denote a family of perfect polyhedral primary partitions of $\Omega$. That is,

$$
\Omega=\bigcup_{K \in T_{n}} K
$$

where $K \in T_{n}$ is a polyhedral and the intersection of any two elements $K_{1}, K_{2} \in T_{n}$ is either an empty set, a common vertex, or a common edge (face). For $K \in T_{n}$, we set

$$
h_{K}:=\operatorname{diam}(K) \text { and } h_{n}:=\max \left\{h_{K}: K \in T_{n}\right\} .
$$

We require that the partitions $T_{n}$ are quasi-uniform (cf. [30]) in the sense that there exist positive constants $c_{1}$ and $c_{2}$ independent of $h_{n}$ such that for all $K \in T_{n}$,

$$
c_{1} h_{n}^{d} \leq \operatorname{vol}(K) \leq c_{2} h_{n}^{d},
$$

where $\operatorname{vol}(K)$ denotes the volume of the element $K$. We also demand the partitions $T_{n}$ to be regular in the usual sense. To explain the regularity condition, we describe the regularity for the triangular partition and the quadrilateral partition in the case when $d=2$. For $d=2$ we set

$$
\rho_{K}:=\sup \{\operatorname{diam}(S): S \text { is a disk contained in } K\},
$$

and let $h_{K}^{\prime}$ denote the length of the smallest side of $K$ and $\Theta(K)$ the set of interior angles of $K$. In particular, we say a triangulation partition $T_{n}$ is regular (cf. [13]), if there is a positive constant $\sigma$ independent of $h_{n}$ such that

$$
\frac{h_{K}^{\prime}}{\rho_{K}} \leq \sigma, \text { for all } K \in T_{n} \text { and for all } n \in \mathbf{N}_{0} .
$$

If $\theta_{K} \in \Theta(K)$ denotes the smallest angle, then (2.5) is equivalent to the following statement that there exists a constant $\theta_{0}>0$ such that $\theta_{K} \geq \theta_{0}$ for all $K \in T_{n}$. Likewise, we say that a quadrilateral partition $T_{n}$ is regular, if there exist positive constants $\sigma$ and $\theta_{0}$ such that for all $K \in T_{n}$, all $n \in \mathbf{N}_{0}$ and all $\theta \in \Theta(K)$,

$$
\frac{h_{K}}{h_{K}^{\prime}} \leq \sigma \quad \text { and } \quad \theta_{0} \leq \theta \leq \pi-\theta_{0} .
$$

Associated with a primary partition $T_{n}$, we construct a dual partition $T_{n}^{*}$ of the domain $\Omega$. The Lagrange interpolation points (or Hermite interpolation points) of 
any element $K \in T_{n}$ are chosen as the nodes of $K$. We denote by $\Omega_{n}$ the set of all the nodes of all elements $K \in T_{n}$. For each $P \in \Omega_{n}$, we construct a control volume (or dual element) $K_{p}^{*}$ which is often a polyhedral surrounding $P$. Usually, we require that any two different control volumes do not overlap in the sense that

$$
\text { meas }\left(K_{P} \cap K_{P^{\prime}}\right)=0 \text {, if } P \neq P^{\prime} .
$$

The union of all control volumes constitutes another partition $T_{n}^{*}$ of $\Omega$ which is called the dual partition of $T_{n}$. In general, we require $T_{n}^{*}$ to be perfect and quasiuniform. There are different constructions of control volumes and dual partitions. For instance, when $\Omega \subset \mathbf{R}^{2}$, we have the barycenter dual partition and the circumcenter dual partition (Voronoi mesh) (cf. 1, 3, 4, 27, 30]). Recently, the dual partition was constructed in [5] using only the control volumes corresponding to the nodes in a true subset of $\Omega_{n}$.

We next describe the trial space associated with the primary partition $T_{n}$ and the test space associated with the dual partition $T_{n}^{*}$. Generally speaking, the trial space $U_{n}$ can be a finite element space on the grid $T_{n}$ and the test space $V_{n}$ is an appropriate piecewise polynomial space on the dual grid $T_{n}^{*}$. For example, when the trial space has a Lagrange type basis, the corresponding test space may be spanned by the characteristic functions of the control volumes on the dual partition $T_{n}^{*}$. When the trial space has a Hermite type basis, the corresponding test space may be spanned by the "generalized" characteristic functions of the control volumes on the dual partition $T_{n}^{*}$, where the generalized characteristic function is zero outside the control volume and within it the polynomial in the term of the Taylor expansion for which the corresponding derivative is used in the construction of the basis function for the trial space. A general construction of the trial space and the test space of the FVM using higher order polynomials was described recently in [9], where geometric conditions on the elements and equivalent morn conditions were given to ensure the resulting FVM bilinear form is bounded and positive definite.

Specifically, the trial space $U_{n}$ can be a conforming or nonconforming Lagrange type or Hermite type finite element space. For simplicity, in this paper we restrict $U_{n}$ to a conforming finite element space defined by

$$
U_{n}:=\left\{u_{n} \in C^{k-1}(\bar{\Omega}):\left.u_{n}\right|_{K} \in \mathbf{P}_{r}, \text { for all } K \in T_{n},\left.\quad u_{n}\right|_{\partial \Omega}=0\right\}
$$

where for a positive integer $r, \mathbf{P}_{r}$ is the space of polynomials of total degree at most $r$. That is, we choose $U_{n}$ as the space of piecewise polynomials of total degree at most $r$ over $T_{n}$, which globally belong to the Sobolev space $H^{k}(\Omega)$ and satisfy the homogeneous boundary condition on $\partial \Omega$. When $k=1, U_{n}$ is a Lagrange finite element space which has the basis $\Phi_{n}:=\left\{\phi_{P}: P \in \Omega_{n}\right\}$ where $\left.\phi_{P}\right|_{K} \in \mathbf{P}_{r}$, for $K \in T_{n}$ and $\phi_{P}\left(P^{\prime}\right)=\delta_{P, P^{\prime}}$, for $P, P^{\prime} \in \Omega_{n}$. When $k \geq 2$, or equivalently some directional derivatives of the functions in $U_{n}$ are continuous, $U_{n}$ is a Hermite finite element space. For simplicity we use the same notation $\Phi_{n}=\left\{\phi_{P}: P \in \Omega_{n}\right\}$ for a basis of $U_{n}$ even when $U_{n}$ is a Hermite finite element space, although in this case $\phi_{P}$ is not necessarily a nodal basis function or $\Omega_{n}$ is not necessarily a set of the nodes in $T_{n}$, but an abstract index set.

The test space $V_{n}$ is defined associated with the dual partition $T_{n}^{*}$ by

$$
V_{n}:=\left\{v_{n} \in L^{2}(\bar{\Omega}):\left.v_{n}\right|_{K^{*}} \in \mathbf{P}_{r^{\prime}} \text {, for all } K^{*} \in T_{n}^{*},\left.v_{n}\right|_{\partial \Omega}=0\right\} .
$$

Usually, we choose $0 \leq r^{\prime}<r$ and require $\operatorname{dim}\left(U_{n}\right)=\operatorname{dim}\left(V_{n}\right)=D(n)$. Note that we allow a test function to have the regularity order lower than that for a trial 
function. Clearly, $V_{n}$ contains piecewise polynomials of total degree $r^{\prime}$ associated with the dual partition $T_{n}^{*}$ and $V_{n} \subset L^{2}(\bar{\Omega})=W^{0,2}(\Omega)$. Since spaces $U_{n}$ and $V_{n}$ have the same dimension, we denote by $\tilde{\Phi}_{n}:=\left\{\psi_{P}: P \in \Omega_{n}\right\}$ a basis for $V_{n}$.

When the basis of the trial space $U_{n}$ is chosen as a Lagrange type, we often let $r^{\prime}=0$ and choose the basis of $V_{n}$ as the characteristic functions on the dual element. Namely, we choose $\tilde{\Phi}_{n}=\left\{\chi_{P}: P \in \Omega_{n}\right\}$ where for each $P \in \Omega_{n}, \chi_{P}$ denotes the characteristic function on the control volume $K_{P}^{*}$. When $U_{n}$ is a Hermite type finite element space, the basis of $V_{n}$ might be chosen as the "generalized" characteristic functions of the dual elements which is zero outside the dual element and within the dual element is the polynomials in the terms of the Taylor expansions for which the corresponding derivatives are used in the construction of the basis function for the trial space.(cf., 9, 27, 30]). For instance, when $\Omega \subset \mathbf{R}^{2}$ and $U_{n}$ is the Zienkiewicz finite element space where the function values and the first partial derivatives at the nodes are used in the construction of the basis, the dual basis is chosen as

$$
\tilde{\Phi}_{n}=\left\{\chi_{P},\left(x_{i}-P_{i}\right) \chi_{P}: P:=\left(P_{1}, P_{2}\right) \in \Omega_{n}, i=1,2\right\},
$$

because the corresponding Taylor polynomial of degree one consists of the constant term and the first degree terms, where $x:=\left(x_{1}, x_{2}\right) \in \mathbf{R}^{2}$. The functions in the set $\tilde{\Phi}_{n}$ are the typical examples of the generalized functions.

To describe the FVM, we introduce the bilinear form for equations (2.2) and (2.3)

$$
a_{n}\left(u, v_{n}\right):=\sum_{K^{*} \in T_{n}^{*}} \int_{K^{*}}(A \nabla u) \cdot \nabla v_{n} d x-\sum_{K^{*} \in T_{n}^{*}} \int_{\partial K^{*}}(A \nabla u) \cdot \mathbf{n} v_{n} d s,
$$

where $u \in H_{0}^{1}(\Omega)$ and $v_{n} \in V_{n}$ and $\mathbf{n}$ is the normal vector outward to $K^{*}$, (cf., 30]). We shall call $a_{n}(\cdot, \cdot)$ the FVM bilinear form. Note that unlike the FEM bilinear form which is independent of the partition, the FVM bilinear form $a_{n}$ depends on the the dual partition $T_{n}^{*}$. Moreover, when $r^{\prime}=0$, the test space reduces to the usual piecewise constant space and $a_{n}$ reduces to

$$
a_{n}\left(u, v_{n}\right)=-\sum_{K^{*} \in T_{n}^{*}} \int_{\partial K^{*}}(A \nabla u) \cdot \mathbf{n} v_{n} d s .
$$

By letting

$$
\left(f, v_{n}\right):=\sum_{K^{*} \in T_{n}^{*}} \int_{K^{*}} f(x) v_{n}(x) d x, \text { for } v_{n} \in V_{n},
$$

the FVM is a numerical scheme to find $u_{n} \in U_{n}$ such that

$$
a_{n}\left(u_{n}, v_{n}\right)=\left(f, v_{n}\right), \quad \text { for all } v_{n} \in V_{n} .
$$

The operator and the matrix resulted from the bilinear form $a_{n}\left(u_{n}, v_{n}\right)$ are both illconditioned. The main purpose of this paper is to study multilevel preconditioning of the resulting operator and the corresponding matrix.

We find it convenient to use an interpolation projector $\Pi_{n}$ from the trial space $U_{n}$ onto the test space $V_{n}$ by

$$
\Pi_{n} u_{n}:=\sum_{P \in \Omega_{n}} \alpha_{P} \psi_{P}, \text { for } u_{n}=\sum_{P \in \Omega_{n}} \alpha_{P} \phi_{P} \in U_{n},
$$

which was originally introduced in [27] (see also, 30]). With this projector, we have that $\Pi_{n} \phi_{P}=\psi_{P}$, for all $P \in \Omega_{n}$. Because spaces $U_{n}$ and $V_{n}$ have the same 
dimension, we observe that $\Pi_{n}$ is invertible. In terms of this operator, the system (2.10) is equivalent to

$$
a_{n}\left(u_{n}, \Pi_{n} w_{n}\right)=\left(f, \Pi_{n} w_{n}\right), \quad \text { for all } w_{n} \in U_{n} .
$$

We remark that unlike the finite element method, the bilinear form $a_{n}\left(\cdot, \Pi_{n} \cdot\right)$ is not symmetric in general, that is,

$$
a_{n}\left(u_{n}, \Pi_{n} w_{n}\right) \neq a_{n}\left(w_{n}, \Pi_{n} u_{n}\right), \quad u_{n}, w_{n} \in U_{n} .
$$

It is this lack of symmetry that requires special attention for the analysis of multilevel preconditioning.

It is well known (cf. [9, 20, 30, 43]) that the convergence of FVM depends on the boundedness and positive definiteness of its bilinear form. In this paper we assume that the bilinear form is bounded and positive definite. Specifically, we assume that there exist positive constants $N$ and $M_{0}$ such that for all $n \geq N$,

$$
\left|a_{n}\left(u_{n}, \Pi_{n} w_{n}\right)\right| \leq M_{0}\left\|u_{n}\right\|_{1}\left\|w_{n}\right\|_{1}, \quad \text { for all } u_{n}, w_{n} \in U_{n} .
$$

The bound (2.13) of the bilinear form for various FVM schemes appeared in [9, 20, 30. 43. We also suppose that there exist positive constants $\gamma_{0}$ and $N$ such that for all $n \geq N$,

$$
a_{n}\left(u_{n}, \Pi_{n} u_{n}\right) \geq \gamma_{0}\left\|u_{n}\right\|_{1}^{2}, \quad \text { for all } \quad u_{n} \in U_{n} .
$$

For the linear FVM, (2.14) is a well-known result (see [1, 3, 27, 29, 30, 48]). Proving (2.14) for the higher order or high-dimensional FVM schemes is a challenging task. It was established in 30, for quadratic and cubic FVMs under some restrictions on the primary triangulation. For the quadratic FVM case, (2.14) was proved in 43 under rather weak conditions on the geometry of the triangles in the partition. General geometric conditions which ensure the validity of (2.13) and (2.14) for higher order FVM schemes for the two-dimensional second order equations was presented recently in 9. The geometric conditions and norm equivalence arguments presented in 9 improve the existing results for the known FVM schemes and provide new results for a large class of FVM schemes which are not known in the literature. Note that the boundedness condition (2.13) and the positive definiteness (2.14) of the bilinear form lead to the error estimate of the FVM solution. For more information on convergence of the FVM, the readers are referred to 9 , 43.

We close this section by providing a two-dimensional quadratic FVM scheme (cf., 9, 30, 43). Suppose that $\Omega \subset \mathbf{R}^{2}$ is a polygonal domain and let $T_{n}$ denote a regular and quasi-uniform triangulation of $\Omega$. That is, $T_{n}$ consists of a finite number of triangle elements $K_{Q}$, where $Q$ is the barycenter of the triangle. We denote by $\bar{\Omega}_{n}, \bar{M}_{n}$ and $\Omega_{n}^{*}$, respectively, the set of the vertices, the set of the midpoints of the common side of two adjacent triangle elements, and the set of the barycenters of the triangle elements in $T_{n}$. Moreover, we set $\stackrel{\circ}{\Omega}_{n}=\bar{\Omega}_{n} \backslash \partial \Omega$ and $\stackrel{\circ}{M}_{n}=\bar{M}_{n} \backslash \partial \Omega$. The dual partition $T_{n}^{*}$ consists of the polygons $K_{P_{0}}^{*}$ surrounding $P_{0} \in \bar{\Omega}_{n}$ and $K_{M}^{*}$ surrounding $M \in \bar{M}_{n}$. The polygons $K_{P_{0}}^{*}$ and $K_{M}^{*}$ are called the dual elements. Figures 1 and 2 illustrate these constructions. Figure 1 gives an example for the construction of $K_{P_{0}}^{*}$, where $P_{0} \in \stackrel{\circ}{\Omega}_{n}, P_{i}, i=1,2, \ldots, 7$, are its adjacent vertices, and that $P_{0 i}$ is a point on the line segment joining $P_{0}$ and $P_{i}$ such that $\overline{P_{0} P_{0 i}}=\frac{1}{3} \overline{P_{0} P_{i}}$, where $\overline{P Q}$ denotes the length of the line segment joining points $P$ and $Q$. We connect $P_{0 i}, i=1,2, \ldots, 7$, successively to form the polygon $K_{P_{0}}^{*}$ which surrounds $P_{0}$. Figure 2 is to illustrate the construction of $K_{M}^{*}$, where 
$M \in \bar{M}_{n}$ is the midpoint of the common side of two adjacent triangle elements $K_{Q_{1}}:=\Delta P_{0} P_{1} P_{2}$ and $K_{Q_{2}}:=\Delta P_{0} P_{1} P_{3}$. We denote by $Q_{12}, Q_{13}, Q_{02}, Q_{03}$ the midpoints of $\overline{P_{01} P_{02}}, \overline{P_{01} P_{03}}, \overline{P_{10} P_{12}}$ and $\overline{P_{10} P_{13}}$, respectively. The polygon $K_{M}^{*}$ surrounding $M$ is obtained by connecting $P_{10}, Q_{03}, Q_{2}, Q_{13}, P_{01}, Q_{12}, Q_{1}, Q_{02}$ and $P_{10}$ successively.

In this special case, the trial space $U_{n}$ is chosen as the space spanned by the Lagrange quadratic elements with respect to the triangulation $T_{n}$. Specifically, for each $P_{0} \in \stackrel{\circ}{\Omega}_{n}$ and $M_{0} \in \stackrel{\circ}{M}_{n}$, the corresponding basis functions are the piecewise quadratic polynomials satisfying the interpolation conditions

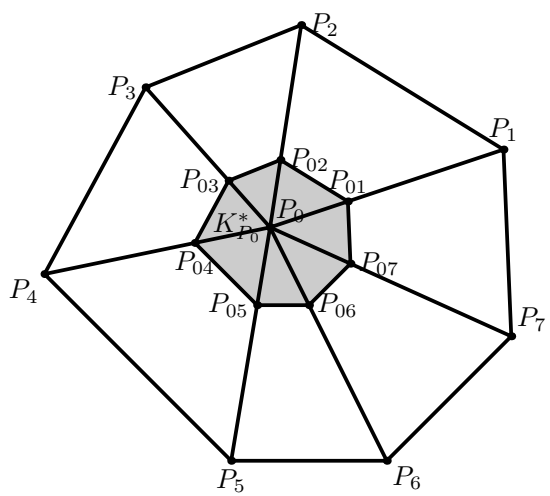

Figure 1. The Dual Element $K_{P_{0}}^{*}$

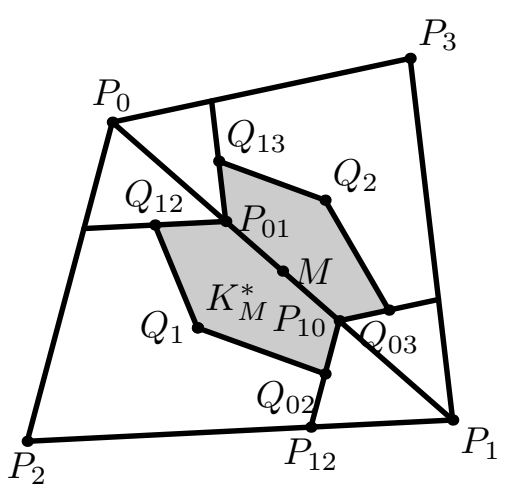

Figure 2. The Dual Element $K_{M}^{*}$

$$
\varphi_{P_{0}}(P)= \begin{cases}1, & P=P_{0} \\ 0, & P \in\left(\bar{\Omega}_{n} \cup \bar{M}_{n}\right) \backslash\left\{P_{0}\right\}\end{cases}
$$

and

$$
\varphi_{M_{0}}(P)= \begin{cases}1, & P=M_{0} \\ 0, & P \in\left(\bar{\Omega}_{n} \cup \bar{M}_{n}\right) \backslash\left\{M_{0}\right\},\end{cases}
$$

respectively. Thus, we have that

$$
U_{n}:=\operatorname{span}\left\{\varphi_{P_{0}}, \varphi_{M_{0}}: P_{0} \in \stackrel{\circ}{\Omega}_{n}, M_{0} \in \stackrel{\circ}{M}_{n}\right\} .
$$

While the test space $V_{n}$ is the piecewise constant function space with respect to the dual partition $T_{n}^{*}$. Namely, for each $P_{0} \in \stackrel{\circ}{\Omega}_{n}$ and $M_{0} \in \stackrel{\circ}{M}_{n}$, the corresponding basis functions are the characteristic functions of $K_{P_{0}}^{*}$ and $K_{M_{0}}^{*}$, respectively; that is, $\psi_{P_{0}}(P)=\chi_{K_{P_{0}}^{*}}(P)$ and $\psi_{M_{0}}(P)=\chi_{K_{M_{0}}^{*}}(P), P \in \Omega$. Hence, we obtain that

$$
V_{n}=\operatorname{span}\left\{\psi_{P_{0}}, \psi_{M_{0}}: P_{0} \in \stackrel{\circ}{\Omega}_{n}, M_{0} \in \stackrel{\circ}{M}_{n}\right\}
$$

The corresponding FVM bilinear form is given by

$$
\begin{aligned}
a_{n}\left(u_{n}, v_{n}\right)= & -\sum_{P_{0} \in \AA_{n}} v_{n}\left(P_{0}\right) \int_{\partial K_{P_{0}}^{*}}\left(A \nabla u_{n} \cdot \mathbf{n}\right) \psi_{P_{0}} d s \\
& -\sum_{M \in \stackrel{\circ}{n}_{n}} v_{n}(M) \int_{\partial K_{M}^{*}}\left(A \nabla u_{n} \cdot \mathbf{n}\right) \psi_{M} d s
\end{aligned}
$$


and the quadratic finite volume scheme for (2.2) and (2.3) is to find $u_{n} \in U_{n}$ such that

$$
a_{n}\left(u_{n}, \psi_{P_{0}}\right)=\left(f, \psi_{P_{0}}\right), \quad P_{0} \in \stackrel{\circ}{\Omega}_{n}, \quad a_{n}\left(u_{n}, \psi_{M}\right)=\left(f, \psi_{M}\right), \quad M \in \stackrel{\circ}{M}_{n} .
$$

\section{Preconditioning of the FVM operator}

This section is devoted to the development of a multilevel preconditioning for the operator induced by the FVM bilinear form $a_{n}$. We will study both two-sided and one-sided preconditioning.

We first introduce the operator corresponding to the bilinear form $a_{n}\left(\cdot, \Pi_{n} \cdot\right)$. The continuity (2.13) of the bilinear form $a_{n}\left(\cdot, \Pi_{n} \cdot\right)$ and the Riesz representation theorem imply that there exists a unique bounded operator $\mathcal{A}_{n}: U_{n} \rightarrow U_{n}$ such that for all $u_{n}, w_{n} \in U_{n}$,

$$
\left(\mathcal{A}_{n} u_{n}, w_{n}\right)=a_{n}\left(u_{n}, \Pi_{n} w_{n}\right) .
$$

We call $\mathcal{A}_{n}$ the FVM operator. Note that, in general, the FVM operator is not self-adjoint due to the nonsymmetry of the bilinear form $a_{n}\left(\cdot, \Pi_{n} \cdot\right)$. It is also clear that when inequality (2.14) holds, the operator $\mathcal{A}_{n}$ is positive definite. Now, by the Riesz representation theorem, we also have $F_{n} \in U_{n}$, such that

$$
\left(F_{n}, w_{n}\right)=\left(f, \Pi_{n} w_{n}\right), \quad \text { for all } w_{n} \in U_{n} .
$$

By (3.1) and (3.2), equation (2.12) has an equivalent operator form

$$
\mathcal{A}_{n} u_{n}=F_{n} .
$$

The main purpose of this section is to precondition the nonself-adjoint operator $\mathcal{A}_{n}$ in the $L_{2}$ norm. To this end, we first present a property of operator $\mathcal{A}_{n}$.

Lemma 3.1. If bilinear form $a_{n}(\cdot, \cdot)$ satisfies hypotheses (2.13) and (2.14), then, for each $n \in \mathbf{N}, \mathcal{A}_{n}$ is bounded from $L_{2}$ to $L_{2}$ and $\mathcal{A}_{n}^{-1}$ exists as a bounded operator from $L_{2}$ to $L_{2}$.

Proof. Since $U_{n}$ is a finite dimensional space, there exist two positive constants $\alpha_{n}, \beta_{n}$, such that

$$
\alpha_{n}\left\|v_{n}\right\|_{0} \leq\left\|v_{n}\right\|_{1} \leq \beta_{n}\left\|v_{n}\right\|_{0}, \text { for all } v_{n} \in U_{n} .
$$

Thus, the $L_{2}$ boundedness of $\mathcal{A}_{n}$ follows directly from inequalities (2.13) and (3.4). The existence of $\mathcal{A}_{n}^{-1}$ in $L_{2}$ is obtained from the inequality (2.14). Moreover, inequalities (2.14) and (3.4) imply the boundedness of $\mathcal{A}_{n}^{-1}$ in $L_{2}$.

We define the condition number of an operator. The operator norm of $\mathcal{B}: U_{n} \rightarrow$ $U_{n}$ induced by the $L_{2}$ norm $\|\cdot\|_{0}$ and the finite dimensional subspace $U_{n}$ is defined by

$$
\|\mathcal{B}\|_{0}:=\sup _{g \in U_{n}} \frac{(\mathcal{B} g, g)}{(g, g)}
$$

When $\mathcal{B}^{-1}: U_{n} \rightarrow U_{n}$ exists as a bounded linear operator, we let $\kappa(\mathcal{B})$ denote the condition number of operator $\mathcal{B}$, i.e., $\kappa(\mathcal{B}):=\|\mathcal{B}\|_{0}\left\|\mathcal{B}^{-1}\right\|_{0}$. Notice that constants $\alpha_{n}$ and $\beta_{n}$ in the proof of Lemma 3.1 may depend on $n$, the condition number of $\mathcal{A}_{n}$ are not necessarily uniformly bounded. This motivates preconditioning $\mathcal{A}_{n}$.

Two sequences of operators, $\mathcal{B}_{n}^{1}$ and $\mathcal{B}_{n}^{2}, n \in \mathbf{N}$, are equivalent, if there exist two positive constants $c_{1}, c_{2}$ such that for all $n \in \mathbf{N}$,

$$
c_{1}\left(\mathcal{B}_{n}^{1} g, g\right) \leq\left(\mathcal{B}_{n}^{2} g, g\right) \leq c_{2}\left(\mathcal{B}_{n}^{1} g, g\right), \quad \text { for all } g \in U_{n} .
$$


When $\mathcal{B}_{n}^{1}$ and $\mathcal{B}_{n}^{2}, n \in \mathbf{N}$, are equivalent, we write $\mathcal{B}_{n}^{1} \sim \mathcal{B}_{n}^{2}$. A common approach to precondition a sequence of operators $\mathcal{A}_{n}$ is to find a sequence of operators $\mathcal{C}_{n}$ such that $\mathcal{C}_{n}^{-1} \sim \mathcal{A}_{n}$.

Lemma 3.2. If bilinear form $a_{n}(\cdot, \cdot)$ satisfies hypotheses (2.13) and (2.14), then the following statements are equivalent.

(i) There exist two positive constants $\gamma \leq \Gamma$ and a sequence of positive definite operators $\mathcal{C}_{n}, n \in \mathbf{N}$, such that

$$
\gamma\left(\mathcal{C}_{n}^{-1} g, g\right) \leq\|g\|_{1}^{2} \leq \Gamma\left(\mathcal{C}_{n}^{-1} g, g\right), \quad \text { for all } g \in U_{n} .
$$

(ii) The sequence $\mathcal{C}_{n}^{-1} \sim \mathcal{A}_{n}$.

Proof. It is easy to conclude from inequalities (2.13), (2.14) and definition (3.1) that

$$
\gamma_{0}\|g\|_{1}^{2} \leq\left(\mathcal{A}_{n} g, g\right) \leq M_{0}\|g\|_{1}^{2}, \quad \text { for all } g \in U_{n}, \quad n \in \mathbf{N} .
$$

Therefore, when (3.6) holds, we have that $\mathcal{C}_{n}^{-1} \sim \mathcal{A}_{n}$ with $c_{1}:=\gamma \gamma_{0}$ and $c_{2}:=M_{0} \Gamma$.

Conversely, we suppose that $\mathcal{C}_{n}^{-1} \sim \mathcal{A}_{n}$. Then, there exist two positive constants $c_{1}, c_{2}$ such that

$$
c_{1}\left(\mathcal{C}_{n}^{-1} g, g\right) \leq\left(\mathcal{A}_{n} g, g\right) \leq c_{2}\left(\mathcal{C}_{n}^{-1} g, g\right), \quad \text { for all } g \in U_{n}, \quad n \in \mathbf{N} .
$$

It follows from (3.7) and (3.8) that

$$
\frac{c_{1}}{M_{0}}\left(\mathcal{C}_{n}^{-1} g, g\right) \leq\|g\|_{1}^{2} \leq \frac{c_{2}}{\gamma_{0}}\left(\mathcal{C}_{n}^{-1} g, g\right), \quad \text { for all } g \in U_{n}, \quad n \in \mathbf{N},
$$

which leads to (3.6) with $\gamma:=\frac{c_{1}}{M_{0}}$ and $\Gamma:=\frac{c_{2}}{\gamma_{0}}$.

As a consequence of the last lemma, we have the following uniform boundedness of the condition numbers of preconditioned operators $\mathcal{C}_{n}^{\frac{1}{2}} \mathcal{A}_{n} \mathcal{C}_{n}^{\frac{1}{2}}$.

Theorem 3.3. Suppose that there exist two positive constants $\gamma \leq \Gamma$ and a sequence of self-adjoint positive definite operators $\mathcal{C}_{n}, n \in N$, such that (3.6) holds. If bilinear form $a_{n}(\cdot, \cdot)$ satisfies hypotheses (2.13) and (2.14), then

$$
\kappa\left(\mathcal{C}_{n}^{\frac{1}{2}} \mathcal{A}_{n} \mathcal{C}_{n}^{\frac{1}{2}}\right) \leq \frac{M_{0} \Gamma}{\gamma_{0} \gamma},
$$

where $\gamma_{0}$ and $M_{0}$ are the positive constants appearing in (2.13) and (2.14), respectively.

Proof. By Lemma 3.2, we have that $C_{n}^{-1} \sim \mathcal{A}_{n}$. That is,

$$
\left(\mathcal{C}_{n}^{-1} h, h\right) \sim\left(\mathcal{A}_{n} h, h\right), \text { for all } h \in U_{n} .
$$

Let $h:=\mathcal{C}_{n}^{\frac{1}{2}} g$. Since $\mathcal{C}_{n}$ are self-adjoint, we observe that

$$
(g, g) \sim\left(\mathcal{C}_{n}^{\frac{1}{2}} \mathcal{A}_{n} \mathcal{C}_{n}^{\frac{1}{2}} g, g\right), \text { for all } g \in U_{n} .
$$

Thus, the condition numbers $\kappa\left(\mathcal{C}_{n}^{\frac{1}{2}} \mathcal{A}_{n} \mathcal{C}_{n}^{\frac{1}{2}}\right)$ are uniformly bounded. By a detailed analysis of the constants in inequalities (2.13), (2.14) and (3.6), we conclude the desired result. 
A result similar to that in Theorem 3.3 for the FEM may be found in [15]; see also [14. In fact, it is the wavelet preconditioning for the FEM that motivates this work.

The preconditioners presented in Theorem 3.3 are two-sided. We next present a one-sided preconditioner for operator $\mathcal{A}_{n}$. To this end, we define the inner product with respect to operator $\mathcal{A}_{n}$. Let $\mathcal{A}_{n}^{*}: U_{n} \rightarrow U_{n}$ denote the adjoint operator of $\mathcal{A}_{n}$ defined by

$$
\left(\mathcal{A}_{n} v, w\right)=\left(v, \mathcal{A}_{n}^{*} w\right), \text { for all } v, w \in U_{n} .
$$

We introduce a self-adjoint operator by setting $\hat{\mathcal{A}}_{n}:=\frac{\mathcal{A}_{n}+\mathcal{A}_{n}^{*}}{2}$. It follows from (2.14) and (2.13) that $\hat{\mathcal{A}}_{n}, n \in \mathbf{N}$, are uniformly bounded and positive definite. We then define the inner product related to $\mathcal{A}_{n}$ by $(v, w)_{\mathcal{A}_{n}}:=\left(\hat{\mathcal{A}}_{n} v, w\right)$. The corresponding norm is defined for all $w \in U_{n}$ by $\|w\|_{\mathcal{A}_{n}}:=\left(\hat{\mathcal{A}}_{n} w, w\right)^{\frac{1}{2}}$ and the corresponding operator norm is defined for $\mathcal{B}: U_{n} \rightarrow U_{n}$ by

$$
\|\mathcal{B}\|_{\mathcal{A}_{n}}:=\sup \left\{\frac{(\mathcal{B} w, w)_{\mathcal{A}_{n}}}{(w, w)_{\mathcal{A}_{n}}}: w \in U_{n}\right\} \text {. }
$$

Note that $\|w\|_{\mathcal{A}_{n}}=\left(\mathcal{A}_{n} w, w\right)^{\frac{1}{2}}$, since $\left(\mathcal{A}_{n} w, w\right)=\left(\hat{\mathcal{A}}_{n} w, w\right)$. Correspondingly, we define the condition number associated with $\mathcal{A}_{n}$ by

$$
\kappa_{\mathcal{A}_{n}}(\mathcal{B}):=\|\mathcal{B}\|_{\mathcal{A}_{n}}\left\|\mathcal{B}^{-1}\right\|_{\mathcal{A}_{n}} .
$$

By (2.13), (2.14) and (3.1), we conclude that $\|w\|_{\mathcal{A}_{n}}$ is equivalent to $\|w\|_{1}$, that is, $\|w\|_{\mathcal{A}_{n}} \sim\|w\|_{1}$.

Lemma 3.4. Let $\mathcal{A}_{n}: U_{n} \rightarrow U_{n}$ be a sequence of positive definite operators. If $\mathcal{C}_{n}: U_{n} \rightarrow U_{n}$ is a sequence of self-adjoint and positive definite operators with $\mathcal{C}_{n}^{-1} \sim \mathcal{A}_{n}$, then

$$
\kappa\left(\mathcal{C}_{n}^{\frac{1}{2}} \mathcal{A}_{n} \mathcal{C}_{n}^{\frac{1}{2}}\right) \sim \kappa_{\mathcal{A}_{n}}\left(\mathcal{C}_{n} \mathcal{A}_{n}\right)
$$

Proof. It suffices to show that

$$
\left\|\mathcal{C}_{n}^{\frac{1}{2}} \mathcal{A}_{n} \mathcal{C}_{n}^{\frac{1}{2}}\right\|_{0} \sim\left\|\mathcal{C}_{n} \mathcal{A}_{n}\right\|_{\mathcal{A}_{n}} \text { and }\left\|\left(\mathcal{C}_{n}^{\frac{1}{2}} \mathcal{A}_{n} \mathcal{C}_{n}^{\frac{1}{2}}\right)^{-1}\right\|_{0} \sim\left\|\left(\mathcal{C}_{n} \mathcal{A}_{n}\right)^{-1}\right\|_{\mathcal{A}_{n}}
$$

We present only a proof for the first relation in 3.13) since the second is proved similarly. Note that $\mathcal{C}_{n}$ and $\mathcal{C}_{n}^{-1}$ are self-adjoint and positive definite. For any $v \in U_{n}$ with $v \neq 0$, we let $w:=\mathcal{C}_{n}^{\frac{1}{2}} v$ and observe that $w \neq 0$. Hence, we have that

$$
\frac{\left\|\mathcal{C}_{n}^{\frac{1}{2}} \mathcal{A}_{n} \mathcal{C}_{n}^{\frac{1}{2}} v\right\|_{0}}{\|v\|_{0}}=\frac{\left\|\mathcal{C}_{n}^{-\frac{1}{2}} \mathcal{C}_{n} \mathcal{A}_{n} w\right\|_{0}}{\left\|\mathcal{C}_{n}^{-\frac{1}{2}} w\right\|_{0}}
$$

Observing that $\mathcal{C}_{n}^{\frac{1}{2}}$ and $\mathcal{C}_{n}^{-\frac{1}{2}}$ are also self-adjoint, we have that

$$
\frac{\left\|\mathcal{C}_{n}^{-\frac{1}{2}} \mathcal{C}_{n} \mathcal{A}_{n} w\right\|_{0}}{\left\|\mathcal{C}_{n}^{-\frac{1}{2}} w\right\|_{0}}=\frac{\left(\mathcal{C}_{n}^{-1} \mathcal{C}_{n} \mathcal{A}_{n} w, \mathcal{C}_{n} \mathcal{A}_{n} w\right)^{\frac{1}{2}}}{\left(\mathcal{C}_{n}^{-1} w, w\right)^{\frac{1}{2}}} .
$$

By the equivalence $\mathcal{C}_{n}^{-1} \sim \mathcal{A}_{n}$, we conclude that

$$
\frac{\left(\mathcal{C}_{n}^{-1} \mathcal{C}_{n} \mathcal{A}_{n} w, \mathcal{C}_{n} \mathcal{A}_{n} w\right)^{\frac{1}{2}}}{\left(\mathcal{C}_{n}^{-1} w, w\right)^{\frac{1}{2}}} \sim \frac{\left(\mathcal{A}_{n} \mathcal{C}_{n} \mathcal{A}_{n} w, \mathcal{C}_{n} \mathcal{A}_{n} w\right)^{\frac{1}{2}}}{\left(\mathcal{A}_{n} w, w\right)^{\frac{1}{2}}}=\frac{\left\|\mathcal{C}_{n} \mathcal{A}_{n} w\right\|_{\mathcal{A}_{n}}}{\|w\|_{\mathcal{A}_{n}}}
$$


where we have used the fact that $\|w\|_{\mathcal{A}_{n}}^{2}=\left(\hat{\mathcal{A}}_{n} w, w\right)=\left(\mathcal{A}_{n} w, w\right)$. From (3.14), (3.15) and (3.16), we obtain that

$$
\frac{\left\|\mathcal{C}_{n}^{\frac{1}{2}} \mathcal{A}_{n} \mathcal{C}_{n}^{\frac{1}{2}} v\right\|_{0}}{\|v\|_{0}} \sim \frac{\left\|\mathcal{C}_{n} \mathcal{A}_{n} w\right\|_{\mathcal{A}_{n}}}{\|w\|_{\mathcal{A}_{n}}}
$$

Therefore, by the definition of the operator norm we conclude the first equation of (3.13).

As a direct consequence of Lemmas 3.2 and 3.4 we have the following result.

Theorem 3.5. If bilinear form $a_{n}(\cdot, \cdot)$ satisfies (2.13) and (2.14) and if there exist positive constants $\gamma \leq \Gamma$ such that the self-adjoint positive definite operators $\mathcal{C}_{n}$, $n \in \mathbf{N}$ satisfy (3.6), then

$$
\kappa_{\mathcal{A}_{n}}\left(\mathcal{C}_{n} \mathcal{A}_{n}\right)=O(1), \quad n \rightarrow \infty .
$$

We now describe a construction of self-adjoint positive definite operator $\mathcal{C}_{n}$ (cf., 15]) which ensures equivalence norm condition (3.6). To this end, we require the primary partition is constructed so that the corresponding trial spaces $U_{n}, n \in N_{0}$, are nested in the sense that

$$
U_{n} \subset U_{n+1} \text {, for all } n \text {. }
$$

We also require that

$$
\overline{\bigcup_{i=0}^{\infty} U_{i}}=L^{2}(\Omega)
$$

However, we do not require test spaces $V_{n}$ to be nested in order to include the commonly used FVM schemes in our setting. Suppose that $Q_{j}, j \in \mathbf{N}_{0}$ is a sequence of linear projectors that maps any $U_{n}, n \geq j$, onto $U_{j}$. For each $j \in \mathbf{N}_{0}$, we define

$$
W_{j+1}:=\left(Q_{j+1}-Q_{j}\right) U_{j+1},
$$

which yields the direct sum decomposition

$$
U_{j+1}=U_{j} \oplus W_{j+1} .
$$

Write $W_{0}:=U_{0}$ so that $U_{n}=\bigoplus_{j=0}^{n} W_{j}, n \in N_{0}$. The corresponding multilevel representation of a $g \in U_{n}$ is given by

$$
g=Q_{0} g+\sum_{j=1}^{n}\left(Q_{j}-Q_{j-1}\right) g .
$$

Define operator $\mathcal{C}_{n}^{-1}: U_{n} \rightarrow U_{n}$ by

$\left(\mathcal{C}_{n}^{-1} g, \tilde{g}\right)=\left(Q_{0} g, Q_{0} \tilde{g}\right)+\sum_{j=1}^{n} 2^{2 j}\left(\left(Q_{j}-Q_{j-1}\right) g,\left(Q_{j}-Q_{j-1}\right) \tilde{g}\right)$, for all $g, \tilde{g} \in U_{n}$

Note that in this case $\mathcal{C}_{n}^{-1}$ is self-adjoint.

We next present a generalization of preconditioner $\mathcal{C}_{n}$. For all $j=0,1, \ldots, n$, let $\mathcal{R}_{j}: U_{j} \rightarrow U_{j}$ be a self-adjoint positive definitive operator satisfying

$$
\left(\mathcal{R}_{j} v_{j}, w_{j}\right) \sim 2^{-2 j}\left(v_{j}, w_{j}\right), \text { for all } v_{j}, w_{j} \in U_{j} .
$$


We define operator $\left(\tilde{\mathcal{C}}_{n}\right)^{-1}: U_{n} \rightarrow U_{n}$ by

$$
\begin{aligned}
& \left(\left(\tilde{\mathcal{C}}_{n}\right)^{-1} g, \tilde{g}\right)=\left(\mathcal{R}_{0}^{-1} Q_{0} g, Q_{0} \tilde{g}\right) \\
& \quad+\sum_{j=1}^{n}\left(\mathcal{R}_{j}^{-1}\left(Q_{j}-Q_{j-1}\right) g,\left(Q_{j}-Q_{j-1}\right) \tilde{g}\right) \text { for all } g, \tilde{g} \in U_{n} .
\end{aligned}
$$

It can be seen that $\left(\tilde{\mathcal{C}}_{n}\right)^{-1} \sim \mathcal{C}_{n}^{-1} \sim \mathcal{A}_{n}$ and thus, $\tilde{\mathcal{C}}_{n}$ can be used to precondition operator $\mathcal{A}_{n}$. Moreover, the results in Theorems 3.3 and 3.5 hold when $\mathcal{C}_{n}$ is replaced by $\tilde{\mathcal{C}}_{n}$.

In particular, when $Q_{j}$ are orthogonal, which ensures (cf. [2, 38]) that

$$
\mathcal{C}_{n}=\sum_{j=0}^{n} 2^{-2 j}\left(Q_{j}-Q_{j-1}\right),
$$

(the operators $\mathcal{C}_{n}$ are the BPX preconditioner introduced in [2]), we choose

$$
\mathcal{C}_{n}^{0}:=\sum_{j=0}^{n} \mathcal{R}_{j} Q_{j} .
$$

It is easily shown that $\mathcal{C}_{n}^{0} \sim \mathcal{C}_{n}$ and thus the results in Theorems 3.3 and 3.5 also hold when $\mathcal{C}_{n}$ is replaced by $\mathcal{C}_{n}^{0}$. The operators $\mathcal{C}_{n}^{0}$ are called parallel subspace correction preconditioners and were used in 2, 38, 40, to precondition the operators induced by the finite element method.

To close this section, we recall the conditions presented in 19 that guarantee hypothesis (3.6) of Theorems 3.3 and 3.5 Let $\omega_{2}(g, t, \Omega)_{2}$ denote the usual second order $L_{2}$ modulus of smoothness of a function $g$ for $l \geq 1$, we also introduce the semi-norm $|\cdot|_{l}$ defined for each $v \in H^{l}(\Omega)$ by

$$
|v|_{l}:=\left(\sum_{|j|=l} \int_{\Omega}\left|D^{j} v\right|^{2}\right)^{\frac{1}{2}} .
$$

Lemma 3.6. Suppose that there exists a positive constant $c$ and $a \tilde{\gamma}>1$ such that for all $n \in N_{0}$ and $g \in U_{n}$,

$$
\omega_{2}(g, t, \Omega)_{2} \leq c \sigma_{n}(t)^{\tilde{\gamma}}\|g\|_{0}, \quad g \in U_{n}
$$

where $\sigma_{n}(t):=\min \left\{1, t 2^{n}\right\}$. Suppose that $Q_{j}, j \in \mathbf{N}_{0}$ are uniformly bounded on $L_{2}(\Omega)$. If there exists a positive constant $c$ such that for all $n \in \mathbf{N}_{0}, v \in H^{l}(\Omega)$,

$$
\inf _{g \in U_{n}}\|v-g\|_{0} \leq c 2^{-n l}|v|_{l},
$$

for some $l>1$, and if $\mathcal{C}_{n}^{-1}$ is defined by (3.21), then there exist positive constants $\gamma, \Gamma$ such that for all $g \in U_{n}, n \in \mathbf{N}_{0}$, inequalities (3.6) hold.

Inequalities (3.26) and (3.27) are called the Bernstein (inverse) estimate and the Jackson (direct) estimate, respectively. In the following, we present conditions on partitions of two-dimensional domains which guarantee the Bernstein and Jackson estimates. For $\Omega \subset \mathbf{R}^{2}$, a triangular partition (or rectangular partition) $T_{n}$ of $\Omega$ is called $\gamma$-quasi-uniform, if there exists a positive constant $\gamma$ such that

$$
\max \left\{\frac{h_{K}}{\rho_{K^{\prime}}}: K, K^{\prime} \in T_{n}\right\} \leq \gamma .
$$


For the triangular partitions, (3.28) is equivalent to (2.4) and (2.5) and for the rectangular partitions, (3.28) is equivalent to (2.4) and the first assumption in (2.6). It is shown in 32 that if for each $n \in \mathbf{N}_{0}, T_{n}$ is $\gamma$-quasi-uniform for some fixed constant $\gamma$, if there exist two positive constants $c_{1}, c_{2}$, such that $c_{1} 2^{-n} \leq$ $h_{n} \leq c_{2} 2^{-n}$, for all $n \in \mathbf{N}_{0}$ and if the trial space $U_{n}, n \in \mathbf{N}_{0}$ are nested, then the Bernstein estimate (3.26) holds with $\tilde{\gamma}=3 / 2$ and the Jackson estimate holds (3.27) with $l:=r+1$.

If the trial spaces $U_{n}, n \in \mathbf{N}_{0}$ have the above described properties, then by Lemma 3.6. Theorems 3.3 and 3.5 hold.

\section{Preconditioning of the FVM matrix}

We discuss in this section the preconditioning of the coefficient matrix of the FVM.

For convenience, we let $\Lambda(n):=\{1,2, \ldots, D(n)\}$ and order the points $P \in \Omega_{n}$ so that the basis $\Phi_{n}$ and $\widetilde{\Phi}_{n}$ defined in Section 2 can be written as $\Phi_{n}=\left\{\phi_{i}: i \in \Lambda(n)\right\}$ and $\widetilde{\Phi}_{n}=\left\{\chi_{i}: i \in \Lambda(n)\right\}$. We introduce the FVM matrix

$$
\mathbf{A}_{n}:=\left[a_{n}\left(\phi_{i}, \chi_{j}\right): i, j \in \Lambda(n)\right]
$$

and let $\mathbf{f}_{n}:=\left[\left(f, \chi_{j}\right): j \in \Lambda(n)\right]$. With these notations, the variational equation (2.10) becomes the linear system

$$
\mathbf{A}_{n} \mathbf{u}_{n}=\mathbf{f}_{n},
$$

where $\mathbf{u}_{n} \in R^{D(n)}$

Note that the matrix $\mathbf{A}_{n}$ induced by the FVM is often nonsymmetric. It is known (cf. 22, 34]) that the general minimal residual method (GMRES) is an efficient scheme for solving a linear system with a nonsymmetric coefficient matrix. Consider the nonsymmetric linear system

$$
\mathbf{G x}=\mathbf{g} .
$$

For the $m$-th approximate solution $\mathbf{x}_{m}$ of (4.2) we define the residual of (4.2) at $\mathbf{x}_{m}$ by

$$
\mathbf{r}_{m}:=\mathbf{g}-\mathbf{G} \mathbf{x}_{m} .
$$

We let $\mu_{1}:=\inf \frac{(\mathbf{G x}, \mathbf{x})}{(\mathbf{x}, \mathbf{x})}$ and $\mu_{2}:=\sup \frac{\|\mathbf{G} \mathbf{x}\|_{0}}{\|\mathbf{x}\|_{0}}$. The following result is due to (cf. [22, 41]).

Lemma 4.1. If $\mu_{1}>0$, then the GMRES method converges and

$$
\left\|\mathbf{r}_{m}\right\|_{0}^{2} \leq\left(1-\frac{\mu_{1}}{\mu_{2}}\right)^{m}\left\|\mathbf{r}_{0}\right\|_{0}^{2}
$$

While using the GMRES method to solve equation (4.1), we have that $\mathbf{G}:=\mathbf{A}_{n}$ and $\mathbf{g}:=\mathbf{f}_{n}$. Thus, the parameters $\mu_{1}$ and $\mu_{2}$ may depend on $n$ and it might happen that $\frac{\mu_{1}}{\mu_{2}} \rightarrow 0$ as $n \rightarrow+\infty$. In this case, the GMRES method either does not converge or converges slowly. For the GMRES method to converge fast while applying it to (4.1) it is necessary to precondition (4.1). We will show that the preconditioners for the finite element matrix also precondition the finite volume matrix for the same boundary value problems.

We first study the preconditioners based on wavelets. For all $j=0,1, \ldots, n$, let $W_{j+1}$ be an orthogonal complement of $U_{j}$ in $U_{j+1}$ which is defined by (3.18). For the index set $\widetilde{\Lambda}(j)$ associated with space $W_{j}$, we let $\Psi_{j}=\left\{\psi_{j, l}: l \in \widetilde{\Lambda}(j)\right\}$ denote 
the wavelet basis for $W_{j}$. Then, $\Psi^{n}=\bigcup_{j=0}^{n} \Psi_{j}$ is the wavelet basis for $U_{n}$. Let $\mathbf{L}$ denote the change of basis matrix from the basis $\Phi_{n}$ to the basis $\Psi^{n}$. That is,

$$
\left(\Psi^{n}\right)^{T}=\Phi_{n}{ }^{T} \mathbf{L}
$$

By introducing

$$
\widetilde{\mathbf{A}}_{n}:=\mathbf{L}^{T} \mathbf{A}_{n} \mathbf{L}, \quad \widetilde{\mathbf{u}}_{n}:=\mathbf{L}^{-1} \mathbf{u}_{n}, \quad \widetilde{\mathbf{f}}_{n}:=\mathbf{L}^{T} \mathbf{f}_{n},
$$

the discrete form (4.1) of the FVM is equivalent to the linear system

$$
\widetilde{\mathbf{A}}_{n} \widetilde{\mathbf{u}}_{n}=\widetilde{\mathbf{f}}_{n} .
$$

In the next theorem we present a preconditioner for the FVM matrix $\mathbf{A}_{n}$ in terms of matrix $\mathbf{L}$.

Theorem 4.2. If bilinear form $a_{n}(\cdot, \cdot)$ satisfied (2.13) and (2.14) with the constants $M_{0}, \gamma_{0}$ and there exist positive constants $\gamma, \Gamma$ and $d_{j, l}, l \in \widetilde{\Lambda}(j), j=0,1, \ldots, n$, such that for each $g \in U_{n}$ with $g:=\sum_{j=0}^{n} \sum_{l \in \widetilde{\Lambda}(j)} y_{j, l} \psi_{j, l}$,

$$
\gamma \sum_{j=0}^{n} \sum_{l \in \widetilde{\Lambda}(j)}\left|d_{j, l} y_{j, l}\right|^{2} \leq\|g\|_{1}^{2} \leq \Gamma \sum_{j=0}^{n} \sum_{l \in \widetilde{\Lambda}(j)}\left|d_{j, l} y_{j, l}\right|^{2},
$$

then, for all $n \in N$,

$$
\kappa\left(\mathbf{D}^{-1} \mathbf{L}^{T} \mathbf{A}_{n} \mathbf{L D}^{-1}\right) \leq \frac{M_{0} \Gamma}{\gamma_{0} \gamma}
$$

where the diagonal matrix $\mathbf{D}:=\left[d_{j, l}: l \in \widetilde{\Lambda}(j), j=0,1, \ldots, n\right]$.

Proof. For each $g, \tilde{g} \in U_{n}$ with $\tilde{g}:=\sum_{j=0}^{n} \sum_{l \in \widetilde{\Lambda}(j)} \tilde{y}_{j, l} \psi_{j, l}$, we have that

$$
a_{n}\left(g, \Pi_{n} \tilde{g}\right)=\mathbf{y}_{n}^{T} \widetilde{\mathbf{A}}_{n} \widetilde{\mathbf{y}}_{n} .
$$

By (2.13) and the second inequality in (4.6), we have that

$$
\left|\mathbf{y}_{n}^{T} \widetilde{\mathbf{A}}_{n} \widetilde{\mathbf{y}}_{n}\right|=\left|a_{n}\left(g, \Pi_{n} \tilde{g}\right)\right| \leq M_{0}\|g\|_{1}\|\tilde{g}\|_{1} \leq M_{0} \Gamma\left\|\mathbf{D} \mathbf{y}_{n}\right\|_{0}\left\|\mathbf{D} \widetilde{\mathbf{y}}_{n}\right\|_{0} .
$$

In other words, we observe that

$$
\left|\left(\mathbf{D} \mathbf{y}_{n}\right)^{T}\left(\mathbf{D}^{-1} \widetilde{\mathbf{A}}_{n} \mathbf{D}^{-1}\right)\left(\mathbf{D} \widetilde{\mathbf{y}}_{n}\right)\right| \leq M_{0} \Gamma\left\|\mathbf{D} \mathbf{y}_{n}\right\|_{0}\left\|\mathbf{D} \widetilde{\mathbf{y}}_{n}\right\|_{0} .
$$

This ensures that

$$
\left\|\mathbf{D}^{-1} \widetilde{\mathbf{A}}_{n} \mathbf{D}^{-1}\right\|_{0} \leq M_{0} \Gamma .
$$

On the other hand, from (2.14) and the first inequality in (4.6), we obtain that

$$
\mathbf{y}_{n}^{T} \widetilde{\mathbf{A}}_{n} \mathbf{y}_{n}=a_{n}\left(g, \Pi_{n} g\right) \geq \gamma_{0}\|g\|_{1}^{2} \geq \gamma_{0} \gamma\left\|\mathbf{D} \mathbf{y}_{n}\right\|_{0}^{2},
$$

that is,

This yields that

$$
\left(\mathbf{D y}_{n}\right)^{T}\left(\mathbf{D}^{-1} \widetilde{\mathbf{A}}_{n} \mathbf{D}^{-1}\right)\left(\mathbf{D} \mathbf{y}_{n}\right) \geq \gamma_{0} \gamma\|\mathbf{D y}\|_{0}^{2}
$$

From (4.8), (4.9) and (4.4), we complete the proof of (4.7). 
We now apply the GMRES method to the preconditioned equation

$$
\mathbf{D}^{-1} \widetilde{\mathbf{A}}_{n} \mathbf{D}^{-1} \mathbf{x}=\mathbf{D}^{-1} \mathbf{f}_{n} .
$$

The next corollary gives a uniform rate of convergence of the method.

Corollary 4.3. If $\mathbf{r}_{m}$ is the residual of equation (4.10) at the $m$-th approximate solution $\mathbf{x}_{m}$, then

$$
\left\|\mathbf{r}_{m}\right\|_{0}^{2} \leq\left(1-\frac{\gamma_{0} \gamma}{M_{0} \Gamma}\right)^{m}\left\|\mathbf{r}_{0}\right\|_{0}^{2}
$$

Proof. By (4.8) and (4.9) in the proof of Theorem 4.2 and the definition of $\mu_{1}$ and $\mu_{2}$, we obtain that $\mu_{1} \geq \gamma_{0} \gamma$ and $\mu_{2} \leq M_{0} \Gamma$. Estimate (4.11) follows directly from these bounds and Lemma 4.1

We remark that condition (4.6) is fulfilled if an additional stability hypothesis for the wavelet basis is satisfied. For each $j \in \mathbf{N}_{0}$, we say that the basis $\Psi_{j}:=$ $\left\{\psi_{j, i}: k \in \widetilde{\Lambda}(j)\right\}$ is stable, if there exist a constant $c_{j}>0$ such that

$$
\left\|\sum_{i \in \widetilde{\Lambda}(j)} y_{j, i} \psi_{j, i}\right\|_{0}^{2} \sim c_{j}^{2} \sum_{i \in \widetilde{\Lambda}(j)}\left|y_{j, i}\right|^{2} .
$$

It was shown in [15] that if $\Psi_{j}$ is stable for all $j \in \mathbf{N}_{0}$ and (3.6) holds for $\mathcal{C}_{n}$ defined in (3.21), then inequality (4.6) holds with $d_{j, i}:=2^{j} c_{j}, j=0,1, \ldots, n, i \in \widetilde{\Lambda}_{j}$.

We now turn our attention to a one-sided matrix preconditioner associated with the operator $\mathcal{C}_{n}^{0}$. For $k \leq n$ we let $\mathbf{E}_{k}$ denote the representation matrix of the nodal basis $\Phi_{k}=\left[\phi_{k, 1}, \ldots, \phi_{k, D(k)}\right]$ of $U_{k}$ in terms of the nodal basis $\Phi_{n}=\left[\phi_{n, 1}, \ldots, \phi_{n, D(n)}\right]$ of $U_{n}$, that is,

$$
\Phi_{k}=\Phi_{n} \mathbf{E}_{k} .
$$

Moreover, let $\mathbf{R}_{k}$ denote the the matrix representation of operator $\mathcal{R}_{k}$ (cf., [38]), and define

$$
\mathbf{C}_{n}^{0}:=\sum_{k=0}^{n} \mathbf{E}_{k} \mathbf{R}_{k} \mathbf{E}_{k}^{T}
$$

Since $\mathbf{C}_{n}^{0}$ is nonsingular, the discrete form (4.1) of the FVM is equivalent to the linear system

$$
\mathbf{C}_{n}^{0} \mathbf{A}_{n} \mathbf{u}_{n}=\mathbf{C}_{n}^{0} \mathbf{f}_{n} .
$$

We define an inner product with respect to $\mathbf{A}_{n}$ by

$$
(\mathbf{v}, \mathbf{w})_{\mathbf{A}_{\mathbf{n}}}:=\left(\hat{\mathbf{A}}_{\mathbf{n}} \mathbf{v}, \mathbf{w}\right) \text {, for all } \mathbf{v}, \mathbf{w} \in R^{D(n)},
$$

where $\hat{\mathbf{A}}_{\mathbf{n}}:=\frac{1}{2}\left(\mathbf{A}_{n}+\mathbf{A}_{n}^{t}\right)$ and $\mathbf{A}_{n}^{t}$ is the transpose of the matrix $\mathbf{A}_{n}$. We also define an induced norm of vector $\mathbf{u} \in R^{D(n)}$ by

$$
\|\mathbf{u}\|_{\mathbf{A}_{n}}:=(\mathbf{u}, \mathbf{u})_{\mathbf{A}_{\mathbf{n}}}^{\frac{1}{2}} .
$$

For a $D(n) \times D(n)$ matrix $\mathbf{A}$, we let $\|\mathbf{A}\|_{\mathbf{A}_{n}}$ denote the matrix norm of $\mathbf{A}$ induced by the vector norm $\|\mathbf{u}\|_{\mathbf{A}_{n}}$. Thus, $\mathbf{A}_{n}$-condition number $\kappa_{\mathbf{A}_{n}}$ is defined accordingly.

We next consider the two-sided preconditioned matrix

$$
\tilde{\mathbf{A}}_{n}:=\left(\mathbf{C}_{n}^{0}\right)^{\frac{1}{2}} \mathbf{A}_{n}\left(\mathbf{C}_{n}^{0}\right)^{\frac{1}{2}} .
$$


Theorem 4.4. If $\mathbf{C}_{n}^{0}$ is the matrix defined by (4.14), then $\kappa\left(\tilde{\mathbf{A}}_{n}\right)=O(1)$.

Proof. For a $\mathbf{u} \in R^{D(n)}$, we let $u_{n}:=\Phi_{n} \mathbf{u}$. By (3.1), we have that

$$
\mathbf{u}^{T} \mathbf{A}_{n} \mathbf{u}=a\left(u_{n}, \Pi_{n} u_{n}\right)=\left(\mathcal{A}_{n} u_{n}, u_{n}\right) .
$$

Let $\mathbf{v}:=\left(\mathbf{C}_{n}^{0}\right)^{-\frac{1}{2}} \mathbf{u}$ and $v_{n}:=\Phi_{n} \mathbf{v}$. Then, $v_{n}=\left(\mathcal{C}_{n}^{0}\right)^{-\frac{1}{2}} u_{n}$, where $\mathcal{C}_{n}^{0}$ is the operator defined by (3.25). Thus, by (4.17), we obtain that

$$
\mathbf{v}^{T} \tilde{\mathbf{A}}_{n} \mathbf{v}=\left(\mathcal{A}_{n}\left(\mathcal{C}_{n}^{0}\right)^{\frac{1}{2}} v_{n},\left(\mathcal{C}_{n}^{0}\right)^{\frac{1}{2}} v_{n}\right)=\left(\left(\mathcal{C}_{n}^{0}\right)^{\frac{1}{2}} \mathcal{A}_{n}\left(\mathcal{C}_{n}^{0}\right)^{\frac{1}{2}} v_{n}, v_{n}\right),
$$

where we have used the fact that $\mathcal{C}_{n}^{0}$ is self-adjoint. It follows from (4.18) that

$$
\kappa\left(\tilde{\mathbf{A}}_{n}\right)=\kappa\left(\left(\mathcal{C}_{n}^{0}\right)^{\frac{1}{2}} \mathcal{A}_{n}\left(\mathcal{C}_{n}^{0}\right)^{\frac{1}{2}}\right) .
$$

Since $\mathcal{C}_{n}^{0} \sim \mathcal{C}_{n}$, Theorem 3.3 is valid when $\mathcal{C}_{n}$ is replaced by $\mathcal{C}_{n}^{0}$. Thus, we have the desired result.

The next lemma shows that the condition number of $\tilde{\mathbf{A}}_{n}$ is equivalent to the $\mathbf{A}_{n}$-condition number of $\mathbf{C}_{n}^{0} \mathbf{A}_{n}$.

Lemma 4.5. If $\mathbf{C}_{n}^{0}$ is the matrix defined by (4.14), then $\kappa_{\mathbf{A}_{n}}\left(\mathbf{C}_{n}^{0} \mathbf{A}_{n}\right) \sim \kappa\left(\tilde{\mathbf{A}}_{n}\right)$.

Proof. By the same arguments used in the proof of Lemma 3.4, we show by a change of variables that

$$
\left\|\tilde{\mathbf{A}}_{n}\right\|_{0} \sim\left\|\mathbf{C}_{n} \mathbf{A}_{n}\right\|_{\mathbf{A}_{\mathbf{n}}} \text { and }\left\|\tilde{\mathbf{A}}_{n}^{-1}\right\|_{0} \sim\left\|\left(\mathbf{C}_{n} \mathbf{A}_{n}\right)^{-1}\right\|_{\mathbf{A}_{\mathbf{n}}} .
$$

Thus, the desired equivalence relation follows.

As a direct consequence of Theorem 4.4 and Lemma 4.5, we have the next result regarding the one-sided preconditioning.

Theorem 4.6. If $\mathbf{C}_{n}^{0}$ is the matrix defined by (4.14), then $\kappa_{\mathbf{A}_{n}}\left(\mathbf{C}_{n}^{0} \mathbf{A}_{n}\right)=O(1)$.

Now we consider solving the preconditioned linear system (4.15) by using the GMRES method. In each iteration step of the GMRES method, we use the inner product $(\cdot, \cdot)_{\mathbf{A}_{\mathbf{n}}}$ instead of the $l_{2}$-inner product. Let $\mathbf{r}_{m}$ denote the residual for equation (4.15) at the $m$-th approximate solution and define

$$
\mu_{1}(n):=\inf \frac{\left(\mathbf{C}_{n}^{0} \mathbf{A}_{n}, \mathbf{x}\right)_{\mathbf{A}_{\mathbf{n}}}}{(\mathbf{x}, \mathbf{x})_{\mathbf{A}_{\mathbf{n}}}} \text { and } \mu_{2}(n):=\sup \frac{\left\|\mathbf{C}_{n}^{0} \mathbf{A}_{n} \mathbf{x}\right\|_{\mathbf{A}_{\mathbf{n}}}}{\|\mathbf{x}\|_{\mathbf{A}_{\mathbf{n}}}} .
$$

The estimate (4.3) can be modified according to the inner product $(\cdot, \cdot)_{\mathbf{A}_{\mathbf{n}}}$ as

$$
\left\|\mathbf{r}_{m}\right\|_{\mathbf{A}_{\mathbf{n}}}^{2} \leq\left(1-\frac{\mu_{1}(n)}{\mu_{2}(n)}\right)^{m}\left\|\mathbf{r}_{0}\right\|_{\mathbf{A}_{\mathbf{n}}}^{2} .
$$

The next corollary shows the uniform convergence rate of the method.

Corollary 4.7. There exists a constant $\rho \in(0,1)$ such that for all $n$,

$$
\left\|\mathbf{r}_{m}\right\|_{\mathbf{A}_{\mathbf{n}}}^{2} \leq(1-\rho)^{m}\left\|\mathbf{r}_{0}\right\|_{\mathbf{A}_{\mathbf{n}}}^{2} .
$$

Proof. By Lemma 4.5 and Theorem 4.4 we have that

$$
\left\|\mathbf{C}_{n}^{0} \mathbf{A}_{n}\right\|_{\mathbf{A}_{n}} \sim\left\|\left(\mathbf{C}_{n}^{0} \mathbf{A}_{n}\right)^{-1}\right\|_{\mathbf{A}_{n}}=O(1) .
$$

Therefore, there exist two constants $\rho_{1}$ and $\rho_{2}$ such that for all positive integer $n$, $\mu_{1}(n) \geq \rho_{1}$ and $\mu_{2}(n) \leq \rho_{2}$. Letting $\rho:=\rho_{1} / \rho_{2}$, the estimate (4.20) is a direct consequence of the above inequalities and (4.19). 
Noticing that when $n$ is large, the GMRES may entail a high computational effort. For this reason, in practice, we use a variant of the GMRES, the GMRES(k) which restarts the iterations after $k$ steps, to reduce the computational cost.

\section{A Linear FVM SCHEME}

In this section, we consider solving (2.1) in the two-dimensional space with discontinuous coefficients. Specifically, we suppose that $\Omega \subset \mathbf{R}^{2}$ and $A(x)=\alpha(x) I$, where $I$ is the $2 \times 2$ identity matrix, $\alpha \in L^{\infty}(\Omega)$ is a piecewise smooth function and there exists a positive $\alpha_{0}>0$ such that $\alpha(x) \geq \alpha_{0}$, for all $x \in \Omega$. We shall prove that the FVM bilinear form using linear elements for the trial space for solving the equation can be expressed as the linear FEM bilinear form for solving the same equation with a small perturbation. As a result, the corresponding FVM matrix can be preconditioned by an efficient preconditioner for the FEM matrix.

We now describe the linear FVM for solving the equation. Let $\mathcal{T}_{n}$ be a quasiuniform, shape regular triangulation of $\Omega$ and $U_{n}$ the associated linear finite element space. We construct the dual partition $\mathcal{T}_{n}^{*}$ with the barycentric mesh described below (see, for example, [9, 30, 43]). The control volume $K_{P}^{*}$ associated with each node $P$ is obtained by connecting successively with lines the midpoints of the adjacent edges of $P$ and the barycenters of the adjacent triangles of $P$. The test space $V_{n}$ consists of the piecewise constant functions with respect to the dual mesh $\mathcal{T}_{n}^{*}$. The corresponding FVM is to find a solution $u_{n} \in U_{n}$ such that equation (2.10) is satisfied with the FVM bilinear form

$$
a_{n}(u, v)=-\sum_{P \in \Omega_{n}} v(P) \int_{\partial K_{P}^{*}}(\alpha \nabla u \cdot \mathbf{n}) \psi_{P} d s, \text { for all } u \in U_{n}, v \in V_{n},
$$

and $\Omega_{n}$ being the set of all vertices in $\mathcal{T}_{n}$ and $\psi_{P}, P \in \Omega_{n}$, are a basis for the test space $V_{n}$.

The finite element bilinear form for the same boundary value problem has the form

$$
a_{e}(u, v)=(\alpha \nabla u, \nabla v), \text { for all } u, v \in U_{n} .
$$

It is well known (see e.g., $1,30,43$ ) that when the coefficient $\alpha$ is piecewise constant with respect to $\mathcal{T}_{n}$, we have the identity

$$
a_{n}(u, v)=a_{e}(u, v), \text { for all } u, v \in U_{n} .
$$

We shall study the more general case when $\alpha$ is piecewise continuous. For this purpose, we introduce the modulus of piecewise continuity

$$
m\left(\alpha, \mathcal{T}_{n}\right):=\sup _{K \in \mathcal{T}_{n}} m(\alpha, K)
$$

where

$$
m(\alpha, K):=\sup _{x, y \in K}|\alpha(x)-\alpha(y)| .
$$

Note that the quantity $m\left(\alpha, \mathcal{T}_{n}\right)$ presents only the variance of $\alpha$ in the interior of elements of $\mathcal{T}_{n}$. It does not depend on the jump of $\alpha$ across edges of $\mathcal{T}_{n}$. Since $\alpha$ is piecewise continuous, we have that

$$
\lim _{n \rightarrow \infty} m\left(\alpha, \mathcal{T}_{n}\right)=0 .
$$


We also introduce the relative modulus of piecewise continuity by setting

$$
m_{r}\left(\alpha, \mathcal{T}_{n}\right):=\sup _{K \in \mathcal{T}_{n}} \frac{m(\alpha, K)}{\alpha_{K}}
$$

where $\alpha_{K}$ is the average of $\alpha$ in $K$ defined by

$$
\alpha_{K}:=\frac{1}{|K|} \int_{K} \alpha(x) d x .
$$

Since $\alpha(x) \geq \alpha_{0}$ for all $x \in \Omega$, we also have

$$
\lim _{n \rightarrow \infty} m_{r}\left(\alpha, \mathcal{T}_{n}\right)=0
$$

The next theorem shows that the FVM bilinear form is a perturbation of the FEM bilinear form.

Theorem 5.1. There exists a positive constant $c$ such that for all positive integers $n$ and for all $v \in U_{n}$,

$$
\left|a_{n}\left(v, \Pi_{n} v\right)-a_{e}(v, v)\right| \leq c m_{r}\left(\alpha, \mathcal{T}_{n}\right) a_{e}(v, v) .
$$

Thus, there exists an integer $N_{0} \in \mathbf{N}$ such that for all $n \geq N_{0}$ and for all $v \in U_{n}$,

$$
\frac{1}{2} a_{e}(v, v) \leq a_{n}\left(v, \Pi_{n} v\right) \leq \frac{3}{2} a_{e}(v, v) .
$$

Proof. For each $K \in \mathcal{T}_{n}$ we define the elementwise FVM bilinear form for $w \in$ $U_{n}, v \in V_{n}$ by

$$
a_{n, K}(w, v):=-\sum_{P \in \Omega_{n} \cap K} v(P) \int_{\partial K_{P}^{*} \cap K}(\alpha \nabla w \cdot \mathbf{n}) \psi_{P} d s,
$$

and the elementwise FEM bilinear form for $u, v \in U_{n}$ by

$$
a_{e, K}(w, v):=\int_{K} \alpha(x) \nabla w \cdot \nabla v d x .
$$

Because $\nabla v$ is constant in each triangle $K \in \mathcal{T}_{n}$, we have that

$$
a_{e, K}(v, v)=\int_{K} \alpha_{K} \nabla v \cdot \nabla v d x .
$$

By the identity (cf. [1, 43])

$$
\int_{K} \nabla v \cdot \nabla v d x=-\sum_{P \in \Omega_{n} \cap K} v(P) \int_{\partial K_{P}^{*} \cap K}(\nabla v \cdot \mathbf{n}) \psi_{P} d s,
$$

we have that

$$
a_{e, K}(v, v)=-\sum_{P \in \Omega_{n} \cap K} v(P) \int_{\partial K_{P}^{*} \cap K}\left(\alpha_{K} \nabla v \cdot \mathbf{n}\right) \psi_{P} d s .
$$

Therefore, for all $v \in U_{n}$,

$$
\begin{aligned}
a_{n, K}\left(v, \Pi_{n} v\right)-a_{e, K}(v, v) & =-\sum_{P \in \Omega_{n} \cap K} v(P) \int_{\partial K_{P}^{*} \cap K}\left(\alpha-\alpha_{K}\right)(\nabla v \cdot \mathbf{n}) \psi_{P} d s \\
& =-\sum_{E \in \mathcal{E}_{n}^{*}} \int_{E \cap K}\left(\alpha-\alpha_{K}\right) \frac{\partial v}{\partial \mathbf{n}}\left[\Pi_{n} v\right] d s
\end{aligned}
$$


where $\mathcal{E}_{n}^{*}$ is the set of interior edges of the dual partition $\mathcal{T}_{n}^{*}$ and $\left[\Pi_{n} v\right]$ is the jump of $\Pi_{n} v$ across the edge $E$. The equation above leads to the inequality

$$
\left|a_{n, K}\left(v, \Pi_{n} v\right)-a_{e, K}(v, v)\right| \leq c m(\alpha, K)|v|_{1, K}^{2}
$$

where the constant $c$ depends only on the minimal angle of $\mathcal{T}_{n}$. Noting that

$$
a_{e, K}(v, v)=\alpha_{K}|v|_{1, K}^{2}, \text { for all } v \in U_{n}
$$

we conclude that

$$
\left|a_{n, K}\left(v, \Pi_{n} v\right)-a_{e, K}(v, v)\right| \leq C m_{r}(\alpha, K) a_{e, K}(v, v) .
$$

Summing the above inequality for all $K \in \mathcal{T}_{n}$, we obtain the desired inequality (5.3).

Finally, using the equation (5.2), we observe that there exists an $N_{0}>0$ such that for all $n \geq N_{0}$,

$$
c m_{r}\left(\alpha, \mathcal{T}_{n}\right) \leq \frac{1}{2}
$$

Thus, the inequality (5.4) is a direct consequence of the above estimate and (5.3).

We remark that the constant $c$ which appeared in the previous theorem depends only on the minimal angle of the triangulation $T_{n}$ and is independent of the jump in the coefficient $\alpha$.

We next discuss the preconditioning of the FVM matrix by making use of the result in the last theorem. It follows from Theorem 5.1 that for sufficiently large $n$, the FVM bilinear form $a_{n}(\cdot, \cdot)$ is equivalent to the FEM bilinear form $a_{e}(\cdot, \cdot)$. This implies that the resulting FEM matrix $\mathbf{A}_{e, n}$ is a good preconditioner for the corresponding FVM matrix $\mathbf{A}_{n}$, and an efficient preconditioner for the FEM matrix $\mathbf{A}_{e, n}$ will be efficient for the precondition of the FVM matrix $\mathbf{A}_{n}$. To this end, we recall an effective preconditioner, proposed in [42, for the FEM matrix $\mathbf{A}_{e, n}$ in the case when the coefficient $\alpha$ has large jumps with respect to $\mathcal{T}_{n}$. Define the mapping $Q_{j}^{\alpha}: H_{0}^{1} \rightarrow U_{n}$ by

$$
\sum_{K \in \mathcal{T}_{n}} \int_{K} \alpha\left(Q_{j}^{\alpha} u\right) v d x=\sum_{K \in \mathcal{T}_{n}} \int_{K} \alpha_{K} u v d x, \text { for all } v \in U_{n} .
$$

The preconditioner is defined by

$$
\mathcal{B}_{n}^{-1}=\sum_{j=0}^{n} \mathcal{R}_{j} Q_{j}^{\alpha}
$$

where $R_{j}$ is the same self-adjoint operator defined by (3.22). In the next theorem, we demonstrate that this preconditioner which was shown in 42 efficient for the FEM matrix $\mathbf{A}_{e, n}$ can also be used for the FVM matrix $\mathbf{A}_{n}$. A similar preconditioner was discussed in 43 .

Theorem 5.2. If $\mathbf{A}_{n}$ and $\mathbf{A}_{e, n}$ denote the stiffness matrices induced by the FVM bilinear form $a_{n}(\cdot, \cdot)$ and the FEM form $a_{e}(\cdot, \cdot)$, respectively, then there exists $N_{0}$ such that for all $n \geq N_{0}$

$$
\kappa\left(\mathbf{A}_{e, n}^{-\frac{1}{2}} \mathbf{A}_{n} \mathbf{A}_{e, n}^{-\frac{1}{2}}\right) \lesssim 1, \quad \kappa_{\mathbf{A}_{n}}\left(\mathbf{A}_{e, n}^{-1} \mathbf{A}_{n}\right) \lesssim 1
$$


Moreover, if $\mathbf{B}_{n}^{-1}$ denotes the matrix for the precondition operator $B_{n}^{-1}$ defined by (5.5), then

$$
\kappa\left(\mathbf{B}_{n}^{\frac{1}{2}} \mathbf{A}_{n} \mathbf{B}_{n}^{\frac{1}{2}}\right) \lesssim\left|\log h_{n}\right|^{2}, \quad \kappa_{\mathbf{A}_{n}}\left(\mathbf{B}_{n} \mathbf{A}_{n}\right) \lesssim\left|\log h_{n}\right|^{2},
$$

where $h_{n}$ is the mesh size of $\mathcal{T}_{n}$.

Proof. The estimates (5.6) are direct consequences of (5.4). It remains to show the estimate (5.7). By Lemma 4.2 in [42, for all $v \in U_{n}$, there holds

$$
\frac{1}{\left|\log h_{n}\right|^{2}}\left(\mathcal{B}_{n}^{-1} v, v\right) \lesssim a_{e}(v, v) \lesssim\left(\mathcal{B}_{n}^{-1} v, v\right)
$$

These inequalities together with (5.4) ensure that for all $v \in U_{n}$,

$$
\frac{1}{\left|\log h_{n}\right|^{2}}\left(\mathcal{B}_{n}^{-1} v, v\right) \lesssim a_{n}\left(v, \Pi_{n} v\right) \lesssim\left(\mathcal{B}_{n}^{-1} v, v\right)
$$

Therefore, by the same arguments used in Section 4, we conclude that the desired estimates (5.7) hold.

\section{Special CASES AND NUMerical EXAmples}

We consider in this section seven special cases on preconditioning the FVM matrices or their GMRES iteration algorithms and present numerical examples to confirm the estimates on condition numbers of the FVM matrices. In the first three cases, we use wavelet preconditioners (in which cases the wavelet bases of the complement space can be easily constructed) and in the last four cases we consider BPX preconditioners. Since Lemma 4.5 ensures that the $\mathbf{A}_{n}$-condition number of the one-sided preconditioned matrix is equivalent to that of its corresponding twosided preconditioned matrix, in this section we shall only consider the two-sided preconditioning. The numerical results presented in this section are all obtained by using Matlab.

\subsection{Wavelet preconditioning.}

\section{Case 1: The Linear FVM Schemes for Two Point Boundary Value Prob- lems.}

In this case and the next two cases, we consider the wavelet precondition. In these cases, the preconditioned matrix is defined as

$$
\tilde{\mathbf{A}}_{n}:=\mathbf{D}^{-1} \mathbf{L}^{T} \mathbf{A}_{n} \mathbf{L} \mathbf{D}^{-1}
$$

where $\mathbf{D}$ is the diagonal matrix which will be defined in the specific context and $\mathbf{L}$ is the change of basis matrix from the nodal basis to the wavelet basis, specifically, in this example, we have that

$$
\mathbf{D}:=\operatorname{diag}\left(d_{j i}: j=0,1, \ldots, n, i \in \tilde{\Lambda}(j)\right) \text {, with } d_{j i}:=2^{j} .
$$

Let $I:=[a, b]$ and $p \in C^{1}(I)$ be a given function with $p_{1} \geq p(x) \geq p_{0}$, for all $x \in I$ and for some positive constants $p_{0}, p_{1}$. Set $L u:=\left(p u^{\prime}\right)^{\prime}$ and for a given function $f \in L^{2}(I)$, we consider the two point boundary value problems

$$
\begin{aligned}
& L u=f \text { in } I, \\
& u(a)=u(b)=0 .
\end{aligned}
$$


The multilevel augmentation method for solving this problem was introduced in [8] and a wavelet preconditioner for the finite element method for this problem was developed in 24 .

For each $n \geq 0$ we introduce a partition $T_{n}$ for $I$ by $a=x_{0}<x_{1}<\ldots<x_{N(n)}=$ $b$. Writing $I_{i}:=\left[x_{i-1}, x_{i}\right]$, the trial space $U_{n}$ is the space of continuous piecewise linear functions on the partition $T_{n}$ satisfying the boundary condition (6.3). Let $\phi_{n, j}$ be a piecewise linear function with knots $x_{i}, i=1,2, \ldots, D(n):=N(n)-1$, satisfying $\phi_{n, j}\left(x_{i}\right)=\delta_{j, i}$, for all $i, j=0,1, \ldots, N(n)$. Then $\Phi_{n}:=\left\{\phi_{n, j}: j \in \Lambda(n)\right\}$ forms a nodal basis for space $U_{n}$, where $\Lambda(n):=\{1,2, \ldots, D(n)\}$. Let $T_{n}^{*}$ be the dual partition of $I$ with nodes given by $a=x_{0}<x_{\frac{1}{2}}<\ldots<x_{N(n)-\frac{1}{2}}<x_{N(n)}=b$, where $x_{i+\frac{1}{2}}=\frac{1}{2}\left(x_{i}+x_{i+1}\right), i=0,1, \ldots, D(n)$. The corresponding dual elements are $I_{0}^{*}=\left[x_{0}, x_{\frac{1}{2}}\right], I_{i}^{*}=\left[x_{i-\frac{1}{2}}, x_{i+\frac{1}{2}}\right], i \in \Lambda(n), I_{N(n)}^{*}=\left[x_{N(n)-\frac{1}{2}}, x_{N(n)}\right]$. For all $j \in \Lambda(n)$, let $\chi_{n, j}$ be the characteristic function of $I_{j}^{*}$. The set of functions $\left\{\chi_{n, j}: j \in \Lambda(n)\right\}$ constitutes a basis for the test space $V_{n}$. In this case, the bilinear form is given by

$$
a\left(u_{n}, \chi_{n, j}\right):=-\left[p\left(x_{j+\frac{1}{2}}\right) u_{n}^{\prime}\left(x_{j+\frac{1}{2}}\right)-p\left(x_{j-\frac{1}{2}}\right) u_{n}^{\prime}\left(x_{j-\frac{1}{2}}\right)\right], \quad j \in \Lambda(n)
$$

and the finite volume matrix is given by $\mathbf{A}_{n}:=\left[a\left(\phi_{n, i}, \chi_{n, j}\right): i, j \in \Lambda(n)\right]$.

To precondition the matrix $\mathbf{A}_{n}$, we first describe a wavelet basis for each of the wavelet spaces $W_{j}$, for $j \geq 1$. For each $j \in \mathbf{N}_{0}$, we let $\widetilde{D}(j):=\operatorname{dim} W_{j}$ and $\widetilde{\Lambda}(j):=\{1,2, \ldots, \widetilde{D}(j)\}$. A pre-wavelet basis of $W_{1}$ is given by (cf., [14, 23] )

$$
\begin{gathered}
\psi_{1,1}=\frac{9}{10} \phi_{1,1}-\frac{3}{5} \phi_{1,2}+\frac{1}{10} \phi_{1,3}, \\
\psi_{1, l}=\frac{1}{10} \phi_{1,2 l-3}-\frac{3}{5} \phi_{1,2 l-2}+\phi_{1,2 l-1}-\frac{3}{5} \phi_{1,2 l}+\frac{1}{10} \phi_{1,2 l+1}, \quad l=2,3, \ldots, N(0)-1, \\
\psi_{1, N(0)}=\frac{1}{10} \phi_{1,2 N(0)-3}-\frac{3}{5} \phi_{1,2 N(0)-2}+\frac{9}{10} \phi_{1,2 N(0)-1} .
\end{gathered}
$$

For each $j>1$, a basis of $W_{j}$ is obtained by the dyadic-dilation and integer-shift of the basis of $W_{1}$. We denote by $\Psi_{j}:=\left\{\psi_{j, l}: l \in \widetilde{\Lambda}(j)\right\}$ the basis of $\bigcup_{l \leq j} W_{l}$.

TABLE 1

\begin{tabular}{r|r|r|r}
\hline$n$ & $N$ & $\kappa\left(\mathbf{A}_{n}\right)$ & $\kappa\left(\tilde{\mathbf{A}}_{n}\right)$ \\
\hline 0 & 3 & 6.1 & 6.1 \\
1 & 7 & 27.5 & 18.5 \\
2 & 15 & 117.1 & 21.8 \\
3 & 31 & 486.7 & 23.5 \\
4 & 63 & 1992.4 & 24.7 \\
5 & 127 & 8084.1 & 26.8 \\
6 & 255 & 32623.0 & 28.3 \\
7 & 511 & 131210.0 & 29.6 \\
\hline
\end{tabular}

Theorem 4.2 ensures that the condition numbers of $\tilde{\mathbf{A}}_{n}$ are uniformly bounded. This result is confirmed by the numerical example with $I=[0,1]$ and $p(x):=1+\sqrt{x}$. Table 1 compares the condition number of $\mathbf{A}_{n}$ with that of $\tilde{\mathbf{A}}_{n}$ at different levels $n$, where $N:=D(n)$ denotes the size of the corresponding matrix. 


\section{Case 2: The Bilinear FVM Schemes on Rectangular Grids for 2-D Laplace Equations.}

We consider (2.2) and (2.3) in a rectangular region $\Omega=[a, b] \times[c, d]$. We choose the trial space $U_{n}$ and the test space $V_{n}$, respectively, as the tensor product space of the univariate trial space and test space on intervals $[a, b]$ and $[c, d]$ described in Example 1. We denote $\Lambda_{1}(n):=\left\{1,2, \ldots, N_{1}(n)-1\right\}$ and $\Lambda_{2}(n):=$ $\left\{1,2, \ldots, N_{2}(n)-1\right\}$ and set $\Lambda(n):=\Lambda_{1}(n) \times \Lambda_{2}(n)$. Let $\phi_{n, j}^{1}, j \in \Lambda_{1}(n)$ and $\phi_{n, j}^{2}, j \in \Lambda_{2}(n)$ be the nodal basis on $[a, b]$ and $[c, d]$, respectively (see Example 1 for the details). For all $(i, j) \in \Lambda(n)$, we define $\phi_{n, i, j}(x, y):=\phi_{n, i}^{1}(x) \phi_{n, j}^{2}(y)$. Then, $\Phi_{n}:=\left\{\phi_{n, i, j}:(i, j) \in \Lambda(n)\right\}$ constitutes a nodal basis for $U_{n}$. For all $(i, j) \in \Lambda(n)$, let $\chi_{n, i, j}$ be the tensor product characteristic functions. Then, $\tilde{\Psi}_{n}:=\left\{\chi_{n, i, j}:(i, j) \in \Lambda(n)\right\}$ constitutes a basis for $V_{n}$. The finite volume matrix is identified with $\mathbf{A}_{n}:=\left[a\left(\phi_{n, k, l}, \chi_{n, i, j}\right),(k, l),(i, j) \in \Lambda(n)\right]$, where

$$
a\left(\phi_{n, k, l}, \chi_{n, i, j}\right)=-\int_{\partial K_{P_{i, j}^{*}}^{*}}\left(A \nabla \phi_{n, k, l}\right) \cdot \mathbf{n} \chi_{n, i, j} d s .
$$

Next we describe the pre-wavelets of $U_{n}$. For each $j \geq 1$, let $W_{j}$ be the orthogonal complement of $U_{j-1}$ in $U_{j}$. The basis of $W_{1}$ is obtained again by the tensor product of the univariate pre-wavelets, that is,

$\psi_{i j}^{1}(x, y):=\phi_{0, i}^{1}(x) \psi_{1, j}^{2}(y), \quad \psi_{i j}^{2}(x, y):=\psi_{1, i}^{1}(x) \phi_{0, j}^{2}(y), \quad \psi_{i j}^{3}(x, y):=\psi_{1, i}^{1}(x) \psi_{1, j}^{2}(y)$.

The pre-wavelet basis of $W_{j}$, for each $j>1$, is obtained by dyadic-dilations and integer-translations of the basis functions for $W_{1}$. Let $\widetilde{\Lambda}(j)$ denote the index set associated with the pre-wavelets basis $\Psi_{j}$ for $W_{j}$ so that $\Psi_{j}:=\left\{\psi_{j, k, l}:(k, l) \in\right.$ $\widetilde{\Lambda}(j)\}$. It is known in [23] that $\widetilde{\Psi}_{j}, j \in \mathbf{N}_{0}$ is a sequence of stable basis. Also, the sequence of orthogonal projectors $\left\{Q_{i}: i \in \mathbf{N}_{0}\right\}$ is uniformly bounded on $L_{2}(\Omega)$. Thus, we have the norm equivalence (4.6) with $d_{j, \mathbf{l}}:=2^{-j}$ for all $\mathbf{l}=\left(l_{1}, l_{2}\right) \in$ $\widetilde{\Lambda}(j)$. With the pre-wavelet basis $\Psi^{(n)}:=\bigcup_{j=0}^{n} \Psi_{j}$ of $U_{n}$, the linear system (4.1) is written in the form (4.5). Let $\mathbf{D}:=\operatorname{diag}\left(d_{j, 1}: \mathbf{1} \in \widetilde{\Lambda}(n)\right)$. Moreover, since the norm equivalence (4.6) holds, by Theorem 4.2, the condition numbers of the preconditioned matrices $\tilde{\mathbf{A}}_{n}$ defined by (6.1) are uniformly bounded. This result is confirmed by the numerical example presented below.

Consider a boundary value problem of the Laplace equation

$$
\begin{cases}-\triangle u(x, y)=f(x, y), & (x, y) \in \Omega:=[0,1] \times[0,1] \\ u(x, y)=0, & (x, y) \in \partial \Omega .\end{cases}
$$

In Table 2 we compare the condition numbers of matrices $\mathbf{A}_{n}$ and $\tilde{\mathbf{A}}_{n}$ at different level $n$, where $N:=D(n)^{2}$ denotes the size of the matrix $\mathbf{A}_{n}$. 
TABLE 2

\begin{tabular}{r|r|r|r}
\hline$n$ & $N$ & $\kappa\left(\mathbf{A}_{n}\right)$ & $\kappa\left(\tilde{\mathbf{A}}_{n}\right)$ \\
\hline 1 & 9 & 3.6 & 3.6 \\
2 & 49 & 13.4 & 25.1 \\
3 & 225 & 52.3 & 31.8 \\
4 & 961 & 207.9 & 35.4 \\
5 & 3969 & 830.4 & 36.4 \\
\hline
\end{tabular}

\section{Case 3: The Linear FVM Schemes on Triangular Grids for 2-D Laplace Equations.}

We consider (2.2) and (2.3) in a convex polygonal domain $\Omega \subset \mathbf{R}^{2}$. Let $T_{n}$ be a triangular partition of $\Omega$, and $T_{n}^{*}$ the corresponding barycenter dual partition (or circumcenter dual partition) of $T_{n}$. We denote by $\bar{\Omega}_{n}$ the set of nodes of $T_{n}$, by $\stackrel{\circ}{\Omega}_{n}=\bar{\Omega}_{n} \backslash \partial \Omega$ the set of the inner nodes and by $\Omega_{n}^{*}$ the set of nodes of the dual partition. Let $K_{Q}$ be the triangular element with barycenter (or circumcenter) $Q \in \Omega_{n}^{*}$ and $K_{P}^{*}$ the dual element surrounding $P \in \bar{\Omega}_{n}$.

The trial space $U_{n}$ is the continuous piecewise linear functions with respect to the partition $T_{n}$ on $\Omega$ satisfying the boundary condition and the test space is the space of piecewise constant functions with respect to the partition $T_{n}^{*}$. Specifically, let $\phi_{n, P}$ be the two-dimensional tent functions satisfying $\phi_{n, P}\left(P^{\prime}\right)=\delta_{P, P^{\prime}}$ for all $P, P^{\prime} \in \stackrel{\circ}{\Omega}_{n}$ and thus, $\Phi_{n}:=\left\{\phi_{n, P}: P \in \stackrel{\circ}{\Omega}_{n}\right\}$ form the nodal basis for $U_{n}$. Moreover, for each $P \in \stackrel{\circ}{\Omega}_{n}$, we denote by $\chi_{n, P}$ the characteristic function of $K_{P}^{*}$. Then, $\tilde{\Psi}_{n}:=\left\{\chi_{n, P}: P \in \stackrel{\circ}{\Omega}_{n}\right\}$ constitutes a basis for $V_{n}$. The finite volume matrix has the form $\mathbf{A}_{n}:=\left[a\left(\phi_{n, P}, \chi_{n, P^{\prime}}\right): P, P^{\prime} \in \stackrel{\circ}{\Omega}_{n}\right]$, where

$$
a\left(\phi_{n, P}, \chi_{n, P^{\prime}}\right)=-\int_{\partial K_{P^{\prime}}^{*}}\left(A \nabla \phi_{n, P}\right) \cdot \mathbf{n} \chi_{n, P^{\prime}} d s .
$$

To precondition $\mathbf{A}_{n}$, we use the two-dimensional pre-wavelets constructed in [35]. Other constructions of two-dimensional pre-wavelets can be found in [18, 25. We now recall the construction. The basis of $W_{0}$ consists of the nodal basis functions of $V_{0}$, i.e., $\psi_{0, P}:=\phi_{0, P}$, for $P \in \stackrel{\circ}{\Omega}_{0}$. For each $0<j \leq n$, we add pre-wavelets corresponding to the new nodal points in the following way. For each $P \in \stackrel{\circ}{\Omega}_{j}$, let $\phi_{j, P}$ denote the nodal basis function on level $j$ corresponding to the point $P$. For each $P \in \stackrel{\circ}{\Omega}_{j-1}$, we define the auxiliary function $\theta_{j, P} \in U_{j}$ by $\theta_{j, P}:=\phi_{j, P}-\frac{1}{8} \phi_{j-1, P}$. For a coarse-grid point $P, \theta_{j, P}$ is a linear combination of a finer grid and coarse-grid nodal basis functions. For $P \in \stackrel{\circ}{\Omega}_{j} \backslash \stackrel{\circ}{\Omega}_{j-1}$, we define

$$
\psi_{j, P}:=\phi_{j, P}-\sum_{P^{\prime} \in I_{j-1}} \frac{\left(\phi_{j, P}, \phi_{j-1, P^{\prime}}\right)_{L_{2}}}{\left(\theta_{j, P}, \phi_{j-1, P^{\prime}}\right)_{L_{2}}} \theta_{j, P^{\prime}}
$$

It is known (cf. 35]) that $\Psi^{(n)}:=\left\{\psi_{j, P}: 0 \leq j \leq n, P \in \stackrel{\circ}{\Omega}_{j} \backslash \stackrel{\circ}{\Omega}_{j-1}\right\}$ constitutes a Riesz basis (with respect to the $L_{2}$ norm ) for $U_{n}$. Let $\mathbf{L}$ denote the change basis matrix between the nodal basis and the pre-wavelet basis and let $\mathbf{D}$ be the diagonal matrix defined as in the last example. By Theorem 4.2, the condition numbers of 
the matrices $\tilde{\mathbf{A}}_{n}$ defined by equation (6.1) are uniformly bounded. We confirm this result by the numerical example, where we consider the boundary value problem (6.5). In Table 3, we compare the condition number of $\mathbf{A}_{n}$ and $\tilde{\mathbf{A}}_{n}$ at a different level $n$. Again in Table 3, $N$ denotes the size of $\mathbf{A}_{n}$.

TABLE 3

\begin{tabular}{l|l|r|c}
\hline$n$ & $N$ & $\kappa\left(\mathbf{A}_{n}\right)$ & $\kappa\left(\tilde{\mathbf{A}}_{n}\right)$ \\
\hline 1 & 9 & 5.8 & 12.2 \\
2 & 49 & 25.3 & 33.2 \\
3 & 225 & 103.1 & 46.2 \\
4 & 961 & 414.3 & 51.8 \\
5 & 3969 & 1659.4 & 54.2 \\
\hline
\end{tabular}

\subsection{BPX-preconditioning.}

Case 4: The Quadratic FVM Schemes on Triangular Grids for 2-D Laplace Equations.

In the next four cases, we use the two-sided BPX-preconditioner

$$
\mathbf{C}_{n}=\sum_{k=0}^{n} \mathbf{E}_{k} \mathbf{D}_{k}^{-1} \mathbf{E}_{k}^{\mathbf{T}}
$$

where $\mathbf{E}_{k}$ is defined in Section 4 and $\mathbf{D}_{k}$ is the diagonal matrix with the diagonal entries equal to the diagonal entries of $\mathbf{A}_{k}$. The resulting preconditioned matrix has the form

$$
\tilde{\mathbf{A}}_{n}:=\mathbf{C}_{n}^{1 / 2} \mathbf{A}_{n} \mathbf{C}_{n}^{1 / 2} .
$$

In this example, we consider the Laplace equation (2.2) and (2.3) in a square region $\Omega:=[0,1] \times[0,1]$ and precondition the FVM schemes described at the end of Section 2 for solving the problem. We choose the trial space $U_{n}$ as the piecewise quadratic finite element space on $T_{n}$ and the test space $V_{n}$ as the piecewise constant space corresponding to the dual partition $T_{n}^{*}$.

In Table 4 we present the computed condition numbers of matrices $\mathbf{A}_{n}$ and $\tilde{\mathbf{A}}_{n}$ : at different levels. The numerical results confirm the uniform boundedness of the condition numbers of the preconditioned matrices, ensured by Theorem 4.4. We solve the corresponding linear algebraic systems by using the GMRES(10). That is, we restart the process after 10 steps in the GMRES iteration. In Table 4, in addition to the condition numbers of the matrices, we also present the iteration numbers for both the unpreconditioned method and the preconditioned method that allow us to fulfill the error tolerance $10^{-6}$. In this section, we use iter0 and iter 1 to denote the iteration numbers for the unpreconditioned and preconditioned FVM schemes, respectively. We observe that the numbers of iterations are bounded above by 37 for different matrix sizes for the preconditioned schemes. This implies that for a given error tolerance, the computational cost for solving the preconditioned linear system is of $\mathcal{O}(n)$. 
TABLE 4

\begin{tabular}{r|r|r|r|r|r}
\hline$n$ & $N$ & $\kappa\left(\mathbf{A}_{n}\right)$ & $\kappa\left(\tilde{\mathbf{A}}_{n}\right)$ & iter0 & iter1 \\
\hline 1 & 9 & 5.8 & 5.5 & 13 & 13 \\
2 & 49 & 25.3 & 9.1 & 26 & 24 \\
3 & 225 & 103.1 & 12.1 & 65 & 29 \\
4 & 961 & 414.3 & 15.0 & 292 & 33 \\
5 & 3969 & 659.4 & 17.6 & 1121 & 36 \\
6 & 16129 & 6639.5 & 19.7 & 4468 & 37 \\
7 & 65025 & 26560.1 & 21.5 & 17754 & 37 \\
\hline
\end{tabular}

\section{Case 5: The linear FVM Schemes for Equations with Discontinuous Coefficients.}

We consider the equation

$$
\begin{cases}-\nabla \cdot(A(x, y) \nabla u(x, y))=f(x, y), & (x, y) \in \Omega, \\ u(x, y)=0, & (x, y) \in \partial \Omega,\end{cases}
$$

where $\Omega:=[0,1] \times[0,1]$ and the coefficient function $A$ is defined as

$$
A(x, y):= \begin{cases}1, & 0 \leq x \leq 1 / 2, \\ \epsilon, & 1 / 2<x \leq 1 .\end{cases}
$$

The linear FVM is used to discretize this equation. In our numerical experiment, the value of $\epsilon$ is chosen to be $0.5,0.1,0.05,0.01,0.005,0.001$. In all these cases, we use the same preconditioner $\mathbf{C}_{n}$ as in Example 4 for different $\epsilon$.

We present in Tables $5 \mathrm{a}$ and $5 \mathrm{~b}$, respectively, the condition numbers of the unpreconditioned matrices $\mathbf{A}$ and the two-sided preconditioned matrices $\tilde{\mathbf{A}}_{n}$ at different levels $n$ for different values of $\epsilon$. Noting that in this example $\mathbf{A}_{n}$ is a symmetric matrix and thus $\kappa_{\mathbf{A}_{n}}\left(\mathbf{C}_{n} \mathbf{A}_{n}\right) \sim \kappa\left(\tilde{\mathbf{A}}_{n}\right)$, the numerical results presented in Table $5 \mathrm{~b}$ also give the condition numbers of the one-sided preconditioning. We observe that for unpreconditioned matrices, the condition numbers increase significantly as the $n$ increases (to infinity) or as the parameter $\epsilon$ decreases (to zero). However, the condition numbers of the preconditioned matrices are uniformly bounded with respect to $n$ and $\epsilon$. These results support our theoretical estimates presented in Section 5 .

TABLE 5a: The condition numbers of the unpreconditioned matrices $\mathbf{A}_{n}$

\begin{tabular}{r|r|r|r|r|r|r|r}
\hline$n$ & $N$ & $\epsilon=0.5$ & $\epsilon=0.1$ & $\epsilon=0.05$ & $\epsilon=0.01$ & $\epsilon=0.005$ & $\epsilon=0.001$ \\
\hline 1 & 9 & 7.2 & 23.5 & 45.6 & 223.2 & 445.3 & 2222.3 \\
2 & 49 & 34.3 & 108.8 & 207.1 & 1000.4 & 1992.9 & 9932.7 \\
3 & 225 & 144.7 & 454.2 & 860.4 & 4137.8 & 8237.8 & 41040.9 \\
4 & 961 & 587.0 & 1838.4 & 3477.6 & 16704.1 & 33250.9 & 165636.1 \\
5 & 3969 & 2357.0 & 7376.4 & 13948.0 & 66978.0 & 133320.0 & 664100.0 \\
\hline
\end{tabular}


TABLE 5b: The condition numbers of the preconditioned matrices $\tilde{\mathbf{A}}_{n}$

\begin{tabular}{c|r|c|c|c|c|c|c}
\hline$n$ & $N$ & $\epsilon=0.5$ & $\epsilon=0.1$ & $\epsilon=0.05$ & $\epsilon=0.01$ & $\epsilon=0.005$ & $\epsilon=0.001$ \\
\hline 1 & 9 & 3.0 & 3.2 & 3.2 & 3.3 & 3.3 & 3.3 \\
2 & 49 & 5.4 & 5.6 & 5.6 & 5.7 & 5.7 & 5.7 \\
3 & 225 & 7.1 & 7.3 & 7.4 & 7.4 & 7.4 & 7.4 \\
4 & 961 & 8.4 & 8.6 & 8.7 & 8.7 & 8.7 & 8.7 \\
5 & 3969 & 9.3 & 9.6 & 9.7 & 9.7 & 9.7 & 9.7 \\
\hline
\end{tabular}

\section{Case 6: The Hermite Element FVM for Two Point Boundary Value Problems.}

We consider solving the two-point boundary value problem

$$
\begin{gathered}
L u=f \quad \text { in } \quad I, \\
u(a)=0, u^{\prime}(b)=0,
\end{gathered}
$$

by using the Hermite cubic element FVM (cf. [30]). In this case, the trial space $U_{n}$ is chosen as the cubic finite element space of the Hermite type. The test space $V_{n}$ is chosen as the piecewise linear function space with basis functions given by

$$
\psi_{0, j}(x)= \begin{cases}1, & x_{j-1 / 2} \leq x \leq x_{j+1 / 2} \\ 0, & \text { elsewhere }\end{cases}
$$

and

$$
\psi_{1, j}(x)= \begin{cases}x-x_{j}, & x_{j-1 / 2} \leq x \leq x_{j+1 / 2} \\ 0, & \text { elsewhere }\end{cases}
$$

The cubic FVM for solving (6.8) and (6.9) is to find $u_{n} \in U_{n}$ such that

$$
\left\{\begin{array}{l}
a\left(u_{n}, \psi_{0, j}\right)=\left(f, \psi_{0, j}\right), j=1,2, \ldots, n, \\
a\left(u_{n}, \psi_{1, j}\right)=\left(f, \psi_{1, j}\right), j=0,1, \ldots, n
\end{array}\right.
$$

We use the two-sided BPX-preconditioners to precondition the linear system above. In Table 6 we present the computed condition numbers of matrices $\mathbf{A}_{n}$ and $\tilde{\mathbf{A}}_{n}$ at different levels, where $\tilde{\mathbf{A}}_{n}$ is defined by (6.7). We report in Table 6 the computed condition numbers of the un-preconditioned and preconditioned matrices, and as well as the iteration numbers for solving the linear system to the accuracy within the tolerance $10^{-6}$ when the GMRES is used. We observe that both the condition numbers of preconditioned matrices and the iteration numbers for preconditioned systems are uniformly bounded. 
TABLE 6

\begin{tabular}{l|r|r|r|r|r}
\hline$n$ & $N$ & $\kappa\left(\mathbf{A}_{n}\right)$ & $\kappa\left(\hat{\mathbf{A}}_{n}\right)$ & iter0 & iter1 \\
\hline 1 & 2 & 58.0 & 2.4 & 4 & 3 \\
2 & 4 & 410.8 & 3.0 & 8 & 7 \\
3 & 8 & 1852.2 & 3.5 & 14 & 10 \\
4 & 16 & 7619.1 & 4.0 & 22 & 11 \\
5 & 32 & 30673.7 & 4.5 & 38 & 13 \\
6 & 64 & 122863.2 & 4.8 & 70 & 14 \\
7 & 128 & 491562.3 & 5.0 & 133 & 14 \\
8 & 256 & 1966240.5 & 5.3 & 261 & 14 \\
9 & 512 & 7864716.8 & 5.4 & 517 & 15 \\
10 & 1024 & 31581495.2 & 5.5 & 1028 & 15 \\
\hline
\end{tabular}

\section{Case 7: The Quadratic FVM Schemes.}

We precondition the quadratic FVM schemes studied in [5]. We again consider the Laplace equation (2.3) and (2.4) in $\Omega:=[0,1] \times[0,1]$. The primal partition $T_{n}$ of the domain is constructed with isosceles right triangles and the corresponding dual partition $T_{n}^{*}$ is the standard barycenter mesh for the linear FVM schemes. The trial space $U_{n}$ is the standard quadratic finite element space on $T_{n}$. The test space $V_{n}$ constitutes the piecewise constant functions on $T_{n}^{*}$ and the quadratic bubble functions at the edges of $T_{n}$. We precondition the resulting linear system with the two-sided BPX-preconditionners $\mathbf{C}_{n}$.

In Table 7 we present the condition numbers of the unpreconditioned matrices $\mathbf{A}_{n}$ and the preconditioned matrices $\tilde{\mathbf{A}}_{n}$ at different levels. We confirm that the condition numbers of the preconditioned matrices are uniformly bounded. Again, the corresponding linear algebraic systems is solved by using the GMRES(10) to accuracy within the error tolerance $10^{-6}$. The iteration numbers are also listed in Table 7.

TABLE 7

\begin{tabular}{r|r|r|r|r|r}
\hline$n$ & $N$ & $\kappa\left(\mathbf{A}_{n}\right)$ & $\kappa\left(\tilde{\mathbf{A}}_{n}\right)$ & iter0 & iter1 \\
\hline 1 & 9 & 4.8 & 4.6 & 14 & 14 \\
2 & 49 & 11.2 & 11.2 & 28 & 27 \\
3 & 225 & 38.7 & 16.4 & 41 & 33 \\
4 & 961 & 150.3 & 20.8 & 97 & 35 \\
5 & 3969 & 596.7 & 24.5 & 416 & 35 \\
6 & 16129 & 2382.4 & 27.5 & 1524 & 36 \\
7 & 65025 & 9525.2 & 30.0 & 5895 & 35 \\
\hline
\end{tabular}

\section{ACKNOWLEDGEMENT}

The authors are grateful to Professor Jinchao Xu for his several intrinsic discussions and valuable suggestions which lead to a significant improvement of this paper. The authors sincerely thank Dr. Junliang Lv, Dr. Dong Mao and graduate student Zhiwen Li of Sun Yat-sen University for their assistance in numerical implementations. 


\section{REFERENCES}

1. R. E. Bank and D. J. Rose, Some error estimates for the box method, SIAM J. Numer. Anal., 24 (1987), 777-787. MR899703 (88j:65235)

2. J. H. Bramble, J. E. Pasciak and J. Xu, Parallel multilevel preconditioners, Math. Comp., 55 (1990), 1-22. MR 1023042(90k:65170)

3. Z. Cai, J. Mandel and S. McCormick, The finite volume element for diffusion equations on general triangulations, SIAM J. Numer. Anal., 28 (1991), 392-402. MR.1087511 (92j:65165)

4. Z. Cai, On the finite volume element method, Numer. Math., 58 (1991), 713-735. MR.1090257 (92d:65188)

5. L. Chen, A new class of high order finite volume methods for second order elliptic equations, SIAM J. Numer. Anal., 47(2010), 4021-4043. MR2585177

6. Z. Chen, The error estimate of generalized difference method 3rd-order Hermite type for elliptic partial differential equations, Northeast. Math. J., 8 (1992), 127-135. MR.1182874 (93e:65125)

7. Z. Chen, R. Li and A. Zhou, A note on the optimal estimate of the finite volume element method, Adv. Comput. Math., 16 (2002), 291-302. MR1894926 (2002m:65113)

8. Z. Chen, B. Wu and Y. Xu, Multilevel augmentation methods for differential equations, Adv. Comput. Math., 24(2006), 213-238. MR2222269 (2007a:65075)

9. Z. Chen, J. Wu and Y. Xu, Higher-order finite volume methods for elliptic boundary value problems, Adv. Comput. Math., to appear.

10. Z. Chen and Y. Xu, The Petrov-Galerkin and iterated Petrov-Galerkin methods for secondkind integral equations, SIAM J. Numer. Anal., 35 (1998), 406-434. MR 1618413 (99h:65214)

11. S. H. Chou and D. Y. Kwak, A covolume method based on rotated bilinears for the generalized Stokes problem, SIAM J. Numer. Anal., 35 (1998), 494-507. MR1618834 (99d:65302)

12. S. H. Chou and D. Y. Kwak, Multigrid algorithms for a vertex-centered covolume method for elliptic problems, Numer. Math., 90 (2002), 441-458. MR.1884225 (2002k:65209)

13. P. G. Ciarlet, The Finite Element Method for Elliptic Problems, North-Holland, Amsterdam, 1978. MR 0520174 (58:25001)

14. W. Dahmen, Wavelet and multiscale methods for operator equations, Acta Numerica 7 (1997), 55-228. MR 1489256 (98m:65102)

15. W. Dahmen and A. Kunoth, Multilevel precorditioning, Numer. Math., 63 (1992), 315-344. MR.1186345 (93j:65065)

16. W. Dahmen, S. Prössdorf, and R. Schneider, Wavelet approximation methods for pseudodifferential equations I: Stability and convergence, Math. Z., 215 (1994), 583-620. MR 1269492 (95g:65148)

17. W. Dahmen, R. Schneider and Y. Xu, Nonlinear functionals of wavelet expansions - Adaptive reconstruction and fast evaluation, Numer. Math. 86 (2000), 49-101. MR.1774010 (2001g:65180)

18. W. Dahmen and R. Stevenson, Element-by-element contruction of wavelets satisfying stability and moment conditions, SIAM J. Numer. Anal. 37(1999), 319-352. MR.1742747(2001c:65144)

19. R. A. DeVore and G. G. Lorentz, Constructive Approximation, Springer-Verlag, Berlin, 1993. MR1261635 (95f:41001)

20. R. Eymard, T. Gallouet, and R. Herbin, Finite Volume Methods, in Handbook of Numerical Analysis VII, North-Holland, Amsterdam, 2000, pp. 713-1020. MR.1804748 (2002e:65138)

21. R. E. Ewing, T. Lin, and Y. Lin, On the accuracy of finite volume element method based on piecewise linear polynomials, SIAM J. Numer. Anal., 39 (2002), 1865-1888. MR.1897941 (2003d:65105)

22. S. C. Eisenstat, H. C. Elman, and M. H. Schultz, Variational iterative methods for nonsymmetric linear systems, SIAM. J. Numer. Anal. 20(1983), 345-357. MR694523 (84h:65030)

23. M. Griebel and P. Oswald, Tensor product type subspace splittings and multilevel iterative methods for anisotropic problems, Adv. Comput. Math., 4 (1995), 171-206. MR1338900 (96e:65069)

24. R.-Q. Jia and S. T. Liu, Wavelet bases of Hermite cubic splines on the interval, Adv. Comput. Math. 25 (2006), 23-39. MR2231693 (2007e:42043)

25. U. Kotyczka and P. Oswald, Piecewise linear pre-wavelets of small Support, in Approximation Theory VIII, Vol 2. Wavelets and Multilevel Approximation, Charles K. Chui and Larry L. Schumaker (eds.), 243-250, World Scientific Publishing Co.,1995. MR.1471789 (98e:42035) 
26. R. Li, Generalized difference methods for two points boundary problem, Acta Scietiarum Naturalium Universitatis Jilineness, 1 (1982), 26-40 (in Chinese).

27. R. Li and P. Zhu, Generalized difference methods for second order elliptic partial differential equations (I), Numer. Math., A Journal of Chinese Univ., 2 (1982), 140-152 (in Chinese).

28. R. Li, Generalized difference methods for a nonlinear Dirichlet problem, SIAM J. Numer. Anal., 24 (1987), 77-88. MR874736 (88c:65091)

29. Y. Li and R. Li, Generalized difference methods on arbitrary quadrilateral networks, J. Comput. Math., 17 (1999), 653-672. MR.1723103 (2000g:65103)

30. R. Li, Z. Chen and $\mathrm{W}$. Wu, The generalized difference methods for differential equations (Numerical analysis of finite volume methods), Marcel Dekker, New York, 2000. MR1731376 (2000j:65003)

31. J. Lv and Y. Li, $L^{2}$ error estimate of the finite volume element methods on quadrilateral meshes, Adv. Comput. Math., 33 (2010), 129-148. MR2659583(2011e:65234)

32. P. Oswald, On function spaces related to finite element approximatin theory, Zeitschrift für Analysis und ihre Anwendungen Bd., 9 (1990), 43-64. MR1063242 (91g:65246)

33. P. Oswald, On discrete norm estimates related to multilevel preconditioners in the finite element method, Constructive Theory of Functions, Varna' 91, Sofia, 1992, 203-214.

34. Y. Saad and M. H. Schultz, GMRES: A generalized minimal residual algorithm for solving nonsymmetric linear systems, SIAM J. Sci. Statist. Comput., 7 (1986), 856-869. MR848568 (87g:65064)

35. R. Stevenson, Locally supported, piecewise polynomial biorthogonal wavelets on non-uniform meshes, Constr. Approx., 19 (2003), 477-508. MR.1998901 (2005a:42032)

36. E. Süli, The accuracy of cell vertex finite volume methods on quadrilateral meshes, Math. Comp., 59 (1992), 359-382. MR.1134740 (93a:65158)

37. Y. Sun, Z. Wang, and Y. Liu, Spectral finite volume method for conservation laws on unstructrured grids VI: Extension to viscous flow, J. Comp. Phys., 215 (2006), 41-58. MR 2215651

38. J. Xu, Iterative methods by space decomposition and subspace correction, SIAM Review, 34 (1992), 581-613. MR:1193013 (93k:65029)

39. J. Xu, An introduction to multigrid convergence theory, in "Iterative Methods in Scientific Computing", (editors: R. Chan, T. Chan and G. Golub), Springer-Verlag, Singapore, 1997. MR 1661962

40. J. Xu, An introduction to multilevel methods, in Wavelets, Multilevel Methods and Elliptic PDEs, 213-302, edited by M. Ainsworth, J. Levesley, M. Marletta, W. A. Light, Oxford Univ. Press, New York, 1997. MR 1600688 (99d:65329)

41. J. Xu and X. Cai, A preconditioned GMRES method for nonsymmetric and indefinite problems, Math. Comp., 59 (1992), 311-319. MR.1134741 (93a:65059)

42. J. $\mathrm{Xu}$, and $\mathrm{Y}$. Zhu, Uniform convergent multigrid methods for elliptic problem with strong discontinuous coefficients, Mathematical Models and Methods in Applied Sciences, 2008, 18, 77-105. MR.2378084 (2008k:65271)

43. J. Xu and Q. Zou, Analysis of linear and quadratic finite volume methods for elliptic equations. Numerische Mathematik, 111(2009), 469-492. MR2470148 (2009i:65199)

44. Y. Xu and Q. Zou, Adaptive wavelet methods for elliptic operator equations with nonlinear terms, Adv. Comput. Math., 19 (2003), 99-146. MR.1973461 (2004f:65201)

45. Y. Xu and Q. Zou, Tree wavelet approximations with applications, Sciences in China Ser. A Math., 48 (2005), 680-702. MR2158483(2006c:42036)

46. H. Yserentant, On the multi-level splitting of finite element spaces, Numer. Math., 49 (1986), 379-412. MR853662 (88d:65068a)

47. H. Yserentant, Two preconditioners based on the multi-level splitting of finite element spaces, Numer. Math., 58 (1990), 163-184. MR.1069277 (91j:65076)

48. P. Zhu and R. Li, Generalized difference methods (finite volume methods) for second order elliptic partial differential equations (II), Numer. Math. A Journal of Chinese Univ., 4 (1982), 360-375 (in Chinese). MR696348 (84m:65116)

49. Q. Zou, Hierarchical error estimates for finite volume approximation solution of elliptic equations, Applied Numerical Mathematics, 60 (2010), 142-153. MR2566084 (2010j:65215) 
Department of Mathematics, Jilin University, Changchun, 130012, People's Republic OF CHINA

E-mail address: yonghai@jlu.edu.cn

School of Mathematical and Computational Sciences, Xiangtan University, Hunan 411105, CHina

E-mail address: shushi@xtu.edu.cn

Department of Mathematics, Syracuse University, Syracuse, New York 13244, and Guangdong Province Key Laboratory of Computational Science, Sun Yat-Sen University, Guangzhou, 510275, People's Republic of China

E-mail address: yxu06@syr.edu

Guangdong Province Key Laboratory of Computational Science, Sun Yat-sen University, Guangzhou, 510275, People's Republic of China.

E-mail address: mcszqs@mail.sysu.edu.cn 